\title{
A nonhomogenizable linear transport equation in $\mathbb{R}^{2}$
}

\author{
ROBERTO PEIRONE
}

\begin{abstract}
In this paper I investigate the homogenizability of linear transport equations with periodic data. Some results on homogenizability and on the form of the limit are known in literature. In particular, in [9], I proved the homogenizability in the two-dimensional case for nonvanishing functions, and, on the other hand I gave an example of a nonhomogenizable equation in the three-dimensional case. In this paper, I describe an example of a nonhomogenizable equation in two dimensions. As in [9], I study the problem using an equivalent formulation in terms of dynamical system properties of the associated ODEs.
\end{abstract}

Mathematics Subject Classification (2000): 35B27 (primary); 37E45 (secondary).

\section{Introduction}

The subject of this paper is that of linear transport equations, and more specially, that of their homogenization. Let us consider the differential problem

$$
\frac{\partial u}{\partial t}(t, x)+f\left(\frac{x}{\varepsilon}\right) \cdot \frac{\partial u}{\partial x}(t, x)=0, \quad u(0, x)=\psi(x) \quad\left(P_{f, \psi}^{\varepsilon}\right)
$$

where $\varepsilon>0, f$ is a $C^{1}$ function from $\mathbb{R}^{N}$ to $\mathbb{R}^{N}$, which is $\mathbb{Z}^{N}$-periodic, i.e., $f(x+m)=f(x)$ for all $m \in \mathbb{Z}^{N}$, and $\psi$ is of class $C^{1}$. Also, $\frac{\partial u}{\partial x}$ denotes the vector $\left(\frac{\partial u}{\partial x_{1}}, \ldots, \frac{\partial u}{\partial x_{N}}\right)$, and $\cdot$ denotes the scalar product in $\mathbb{R}^{N}$. The equation in $P_{f, \psi}^{\varepsilon}$, denoted by $P_{f}^{\varepsilon}$, is called linear transport equation and by homogenization of $P_{f}^{\varepsilon}$ we mean in some sense, to find a sort of limit, for $\varepsilon \rightarrow 0^{+}$, of its solutions.

If $N=1$ it is known and simple to prove that the solutions are strongly convergent and if in addition, $f$ does not vanish, we easily get a limit equation. However, the strong convergence appears to be a very strong requirement in the general case. In fact, if $N>1$ the solutions may not strongly converge even in simple and natural cases ( $c f$. [3, Remark 2.2], and references therein). On the contrary, a sort of weak convergence appears to be a natural requirement. So, we are lead to say that $P_{f}^{\varepsilon}$ is homogenizable if the solutions $u_{\varepsilon, f, \psi}$ of $P_{f, \psi}^{\varepsilon}$ are convergent in the weak* topology of $L_{l o c}^{\infty}$ for every $\psi$ of class $C^{1}$.

Received April 20, 2007; accepted in revised form September 3, 2008. 
Y. Brenier [1] proved the homogenizability of $P_{f}^{\varepsilon}$, under the hypothesis that the divergence of $f$ is 0 . Under the same hypothesis, in other papers, the form of the limit of the solutions has been also investigated. For example, T. Hou and X. Xin in [5] treated the case where $N=2$ and nonvanishing $f$, W.E [4] studied the problem in a much more general context, T. Tassa [10] studied the more general case $\operatorname{div}(p f)=0$ for some $p: \mathbb{R}^{2} \rightarrow \mathbb{R}$, positive, of class $C^{1}$, and periodic. In this paper, I merely consider the problem of the existence of the limit. E. De Giorgi conjectured that $P_{f}^{\varepsilon}$ is homogenizable independently of $f$ [3, Conjecture 1.1]. I previously investigated in [9] the homogenizability problem. The main results in [9] state that in the case $N=2, f(x) \neq 0$ for every $x \in \mathbb{R}^{2}$ (with no conditions on the divergence of some function related to $f$ ), $P_{f}^{\varepsilon}$ is homogenizable, and on the other hand, there exists an example with $N=3, f$ of class $C^{\infty}, f(x) \neq 0$ for every $x \in \mathbb{R}^{3}$, in which $P_{f}^{\varepsilon}$ is not homogenizable. The case $N=2, f$ vanishing somewhere is not covered by the previous results. In [9, Remark 4.11], in fact, an example of $\bar{f}$ such that $P \frac{\varepsilon}{f}$ is not homogenizable in the two-dimensional case, is stated to exist but it is not described. Of course, such a function $f$ has to attain the value 0 somewhere.

In this paper I will describe such an example. I will use a similar notation as in [9], in particular, for $k \in \mathbb{N} \cup\{\infty\}, C^{k}(\mathbb{T})$ denotes the set of functions $f$ of class $C^{k}$ from $\mathbb{R}^{N}$ to $\mathbb{R}^{N}$ which are $\mathbb{Z}^{N}$-periodic. If $f \in C^{1}(\mathbb{T}), x \in \mathbb{R}^{N}$, then $T_{f}^{t}(x)$, or simply $T^{t}(x)$, denotes the value attained at $t$ by the solution of

$$
\left\{\begin{array}{l}
y^{\prime}(t)=f(y(t)) \\
y(0)=x .
\end{array}\right.
$$

We will always mean $N=2$ except in Section 2, where $N$ can also amount to 1 . Let us denote by $E_{f}$ the ODE in the previous formula. In view of [9, Lemma 2.2], which in turn is a simple variant of results in [8], the problem of homogenizability can be seen as a dynamical system problem. More precisely, in order to construct the claimed example, it suffices to find a function $\bar{f} \in C^{1}(\mathbb{T})$ such that the limit

$$
\lim _{t \rightarrow+\infty} \int_{[0,1]^{2}} \frac{T_{\bar{f}, 2}^{t}(x)-x_{2}}{t} d x
$$

does not exist, where $T_{\bar{f}, 2}^{t}$ denotes the second component of $T_{\frac{f}{f}}^{t}$. Note that the function $V_{a}(t, x)=\frac{T_{f}^{t}(x)-x}{t}$ represents the average velocity in a time-interval of length $t$ of a solution of $E_{f}$ starting from $x$. In particular, $V_{a}(t, x)$ cannot pointwise converge for $t \rightarrow+\infty$, and in fact cannot converge a.e., and thus our problem is related to the notion of rotation set introduced in [7]. The existence of a function with such a property is not completely trivial, although I guess it is known. However, in view of (1.1), we have to find a function with the stronger property that the average of $V_{a}(t, x)$ in $x \in[0,1]^{2}$ does not converge for $t \rightarrow+\infty$. This is a similar idea to 
that of the example in the three-dimensional case discussed in [9]. However, the construction of the example in the two-dimensional case is much harder, due to the fact that in two dimensions a solution of $E_{\bar{f}}$ is forced to remain in a "strip" bounded by two solutions (see [9, Proof of Theorem 3.1]), so that it is not trivial to avoid that it approaches a periodic solution. Note that, in spite of what I announced in [9], we can choose the function $\bar{f}$ to be of class $C^{\infty}$ (see Remark 6.3 here).

The function $\bar{f}$ is defined in terms of two functions $\bar{g}$ and $\bar{\Phi}$, the former denoting the section map for the flow on the torus on the manifold $y=0$, and the latter being comparable to the time of the return ( $c f$. for example [6, Section 0.3]). The map $\bar{g}$ is a diffeomorphism of the circle, and is constructed as the limit of a Cauchy sequence of functions in the $C^{1}$ metric, and the approximating functions are defined recursively and their rotation numbers are rational numbers that approximate very quickly the irrational rotation number of $\bar{g}$. Such a method is called Fast-approximation method in [6, Section 12.6], where is used, for example, in the construction of diffeomorphisms of the circle with irrational rotation numbers and pathological conjugacies ( $c f$. also [2, Chapter 3, Section 5]). Sections 2 and 3 describe the plan of the construction. Section 4 introduces a class of functions basic in this construction. In Section 5, the key lemma (Lemma 5.5) is stated, which allows us to construct the $(n+1)^{\text {th }}$ function in terms of the $n^{\text {th }}$ function in the sequence approximating $\bar{g}$. In Section 6, assuming Lemma 5.5, I construct our example. Sections 7 to 9 are devoted to prove Lemma 5.5.

\section{Motivation of the construction}

I start this section by recalling the main definitions concerning the homeomorphisms of the circle. The reader can refer, for example, to [2] and to [6] for information on this topic. Put

$D^{0}(\mathbb{T}):=\{g: \mathbb{R} \rightarrow \mathbb{R}: g-I d$ is 1 -periodic, $g$ strictly increasing and continuous $\}$

If $g \in D^{0}(\mathbb{T})$, then there exists $\rho \in \mathbb{R}$ so that

$$
\frac{g^{n}(x)-x}{n} \underset{n \rightarrow+\infty}{\longrightarrow} \rho
$$

uniformly with respect to $x \in \mathbb{R}$. Such a number $\rho$ is called the rotation number of $g$. Clearly, such a type of map $g$ can be interpreted as a homeomorphism of the circle, where the circle can be defined as $\mathbb{R}$ quotiented by the equivalence relation that identifies two real numbers $a$ and $b$ iff $a-b$ is integer. The simplest map having $\rho$ as its rotation number is $x \mapsto x+\rho$. I recall the following results.

Lemma 2.1. If $p \in \mathbb{Z}, q \in \mathbb{N} \backslash\{0\}$, we have

i) $\rho(g)=\frac{p}{q}$ if and only if there exists $x \in \mathbb{R}$ such that $g^{q}(x)=x+p$. 
ii) If $\rho(g)=\frac{p}{q}$, then for every $x \in \mathbb{R}$ there exists $x^{\prime} \in \mathbb{R}$ such that $g^{n q}(x)-$ $n p \underset{n \rightarrow+\infty}{\longrightarrow} x^{\prime}$, and $g^{q}\left(x^{\prime}\right)=x^{\prime}+p$.

iii) If for some $x \in \mathbb{R}$ we have $g^{q}(x) \geq x+p$ (respectively $g^{q}(x) \leq x+p$ ), then $\rho(g) \geq \frac{p}{q}$ (respectively $\left.\rho(g) \leq \frac{p}{q}\right)$.

iv) If $r \in \mathbb{R}$ and $g^{q}(x)>x+r$ (respectively $g^{q}(x)<x+r$ ) for every $x \in \mathbb{R}$, then $\rho(g)>\frac{r}{q}$ (respectively $\left.\rho(g)<\frac{r}{q}\right)$.

Thus, a map $g \in D^{0}(\mathbb{T})$ has periodic points (interpreted as a map on the circle) if and only if its rotation number is rational, and, in such a case, in some sense, every point is "asymptotically periodic". In the next lemma and in the rest of this paper, $\operatorname{Fr}(a)$ will denote the fractional part of $a \in \mathbb{R}$, i.e., $a-[a]$.

Lemma 2.2. Given $p \in \mathbb{Z}, q \in \mathbb{N} \backslash\{0\}$ with $p$ and $q$ relatively prime, suppose $\rho(g)=\frac{p}{q}$, and put $0_{h}=\operatorname{Fr}\left(g^{h}(0)\right)$. Then, the points $0_{h}, h=0, \ldots, q-1$ are mutually distinct and their order is independent of the particular map having the above properties (once $p$ and $q$ are given).

Hence, taking the map $x \mapsto x+\frac{p}{q}$, we have $0_{h}<0_{h^{\prime}}$ if and only if $\operatorname{Fr}\left(\frac{h p}{q}\right)<$ $\operatorname{Fr}\left(\frac{h^{\prime} p}{q}\right)$.

Note that, as simple examples show, the behaviour of a map in $D^{0}(\mathbb{T})$ is not characterized by its rotation number. For example the map $x \mapsto x+\frac{p}{q}$ has the property that its $q^{\text {th }}$ iterate has the form $x \mapsto x+p$, while for other maps having rotation number equal to $\frac{p}{q}$ too, their iterates only have isolated periodic points on the circle. However, under some relatively mild hypothesis, this does not happen when the rotation number is irrational. This is the statement of the following well known theorem (Denjoy's Theorem).

Theorem 2.3. Suppose that a map $g \in D^{0}(\mathbb{T})$ has the irrational rotation number $\rho$. Suppose further that it is of class $C^{2}$ and has positive derivative. Then $g$ is topologically conjugate to the map $g_{\rho}$ defined as $g_{\rho}(x)=x+\rho$. In other words there exists $\phi \in D^{0}(\mathbb{T})$ such that $g=\phi^{-1} \circ g_{\rho} \circ \phi$.

Note that in general even if $g$ is of class $C^{\infty}$ or even analytic, the conjugacy $\phi$ is not necessarily regular (neither of class $C^{1}$ ). The regularity of $\phi$ depends on arithmetic properties of $\rho$, in particular the map $\phi$ has been proved to be regular when $g$ is sufficiently regular and $\rho$ is a Diophantine number, that roughly speaking means that it cannot be approximated too fast by rationals. I refer to [2] or to [6] for more details.

When we have a differential equation $\left(E_{f}\right)$ as in Introduction, but with $f$ : $\mathbb{R} \rightarrow \mathbb{R}$ 1-periodic, we can define the rotation number of $\left(E_{f}\right)$ as $\lim _{t \rightarrow+\infty} \frac{y(t)}{t}$ where $y$ is any solution of $\left(E_{f}\right)$, or in other words, $\lim _{t \rightarrow+\infty} \frac{T_{f}^{t}(x)}{t}$ where $x \in \mathbb{R}$. It amounts to the rotation number of the Poincaré map of $\left(E_{f}\right)$, which is the map $T_{f}^{1}$. 
If, instead of the 1-dimensional case, we consider maps from $\mathbb{R}^{2}$ to $\mathbb{R}^{2}$ of the form Id plus a $\mathbb{Z}^{2}$ periodic function the situation is more complicated. In general, the limit in (2.1) does not necessarily exist, and even when exists, it can depend on $x$. An example of this type is the differential equation $\left(E_{f}\right)$ where $f(x)$ has the form $\left(g\left(x_{2}\right), 0\right)$. In such a case, a simple argument shows that $\frac{T_{f}^{t}(x)}{t} \underset{t \rightarrow+\infty}{\longrightarrow}\left(g\left(x_{2}\right), 0\right)$. In view of what stated in Introduction, we have in fact to find $f$ such that it is false that the limit of the integrand in (1.1) exists a.e. In order to better understand the construction of next sections, I here describe the difficulties in obtaining it, in particular I will explain why simpler constructions fail. Recall that we have to define a function $\bar{f} \mathbb{Z}^{2}$-periodic and of class $C^{1}$ such that $P_{f}^{\varepsilon}$ is not homogenizable.

For simplicity, I will only consider $\bar{f}$ such that at any point $x \in \mathbb{R}^{2}$ we have

$$
\text { either } \bar{f}_{2}(x)>0 \text { or } \bar{f}(x)=0
$$

so that every solution of $E_{\bar{f}}$, except for those passing through (or approaching) zeroes of $\bar{f}$, tend to $+\infty$ in the second component. Suppose for the moment that $\bar{f}_{2}(x)>0$ for every $x$. Since $\bar{f}$ never vanishes, we know from the results of [9] that $\frac{T_{f}^{t}(x)}{t}$ tends to a (finite) limit as $t \rightarrow+\infty$, for every $x$. The reason for which this happens can be roughly explained in the following way: the solution passing through $(x, 0)$ will reach a point $(\bar{g}(x), 1)$ after a time $\bar{\Psi}(x)$. The map $\bar{g}$ has a rotation number $\bar{\rho}$. Now, putting $\Sigma_{n}(x)=\sum_{i=0}^{n-1} \bar{\Psi}\left(\bar{g}^{i}(x)\right)$ for $n=1,2, \ldots$, we have that for every $x \in \mathbb{R}$, there exists $c_{x} \in \mathbb{R}$ such that

$$
\frac{1}{n} \Sigma_{n}(x) \underset{n \rightarrow+\infty}{\longrightarrow} c_{x}
$$

To deduce (2.3) note that, if $\bar{\rho} \in \mathbb{Q}$, then this follows from Lemma 2.1ii. If, on the contrary, $\bar{\rho}$ is irrational, and for simplicity we suppose we are in the hypothesis of Denjoy's Theorem, we have for some $\phi \in D_{0}(\mathbb{T})$,

$$
\frac{1}{n} \Sigma_{n}(x)=\frac{1}{n} \sum_{i=0}^{n-1} \bar{\Psi} \circ \phi^{-1}(\phi(x)+i \bar{\rho})
$$

that tends to $\int_{[0,1]} \bar{\Psi} \circ \phi^{-1}$. In general, for any $\bar{\rho}$, rational or irrational, it is not difficult to deduce from (2.3) that

$$
\frac{T_{\frac{t}{f}}(x)}{t} \underset{t \rightarrow+\infty}{\longrightarrow} \frac{1}{c_{x}}(\bar{\rho}, 1) .
$$

Return now to the more general case where (2.2) holds. In this case we can mimic the previous considerations. Clearly, for $x$ such that the trajectory through $(x, 0)$ meets a zero of $\bar{f}$, whose $y$-coordinate belongs to $[0,1[, \bar{g}$ is not defined and $\bar{\Psi}$ 
could only be defined to be $+\infty$. I will call those $x$ 1-singular and the other 1regular. So, we have to slightly modify our program. Suppose for simplicity $\bar{f}$ only attains the value 0 on $[0,1[\times[0,1[$ at $(0,0)$ and $\bar{g}$, defined as above on the regular points, can be extended to a sufficiently regular diffeomorphism on $\mathbb{R}$. In this case (2.3) is still valid when $\bar{\rho}$ is rational, by essentially the same argument as in the previous case. When $\bar{\rho}$ is irrational, it is not so simple to extend the argument of the previous case. However, we can use the Birkhoff ergodic Theorem, to conclude that, if $\bar{\Psi} \circ \phi^{-1}$ is in $L^{1}$, then the limit in (2.4), putting $y$ in place of $\phi(x)$, exists for almost all $y$ with respect to Lebesgue measure $\mu$. This implies that the limit in (2.4) exists $\mu$-a.e. provided the map $\phi$ is regular, and this happens, as told above, if $\bar{\rho}$ is Diophantine. It is possible and not very difficult to prove that the case where $\bar{\Psi} \circ \phi^{-1}$ is not in $L^{1}$ can be reconducted to the previous considerations.

In conclusion, in order to construct our counterexample, the rotation number $\bar{\rho}$ of $\bar{g}$ need be an irrational Liouville (that is, not Diophantine) number. First of all, I will construct $\bar{f}$, by using a sort of inverse process, in other words, I will construct two functions $\bar{g}$ and $\bar{\Psi}$ from $\mathbb{R}$ to $\mathbb{R}$, and then, basing on them, I will recover $\bar{f}$. In other words, I use the standard idea of defining a continuous dynamical system in terms of a discrete dynamical system. In order to avoid the value $+\infty$, in place of $\bar{\Psi}$ it would be better to define its reciprocal. However, for technical reasons, I prefer to define a function $\bar{\Phi}$ representing the second component of the velocity $\bar{f}_{2}$ of the solution of $E_{\bar{f}}$ at a point related to $(x, 0)$. Then, I will construct $\bar{f}$ in such a way that $\bar{\Phi}$ is comparable with $\frac{1}{\bar{\Psi}}$, say

$$
\frac{1}{2 \bar{\Psi}} \leq \bar{\Phi} \leq \frac{1}{\bar{\Psi}}
$$

\section{Plan of the construction}

In this section, I describe the general idea behind the construction of the pair $(\bar{g}, \bar{\Phi})$. First of all, I introduce the set of functions suitable to this aim.

Definition 3.1. Let $D^{1}(\mathbb{T})$ be the space $\left\{g \in D^{0}(\mathbb{T}), g\right.$ of class $\left.C^{1}, g^{\prime}>0\right\}$.

We equip $D^{1}(\mathbb{T})$ with a metric $d_{1}$ defined by $d_{1}\left(g_{1}, g_{2}\right)=\sup \left\{\left|g_{1}(x)-g_{2}(x)\right|\right.$, $\left.\left|g_{1}^{\prime}(x)-g_{2}^{\prime}(x)\right|: x \in \mathbb{R}\right\}$. The following result will be used in the sequel.

Lemma 3.2. The map $g \mapsto g^{n}$ from $D^{1}(\mathbb{T})$ to $D^{1}(\mathbb{T})$ is continuous for every $n \in \mathbb{Z}$.

For some consideration it is also useful to equip $D^{1}(\mathbb{T})$ with the metric $d_{0}\left(g_{1}, g_{2}\right)=$ $\sup \left\{\left|g_{1}(x)-g_{2}(x)\right|: x \in \mathbb{R}\right\}$. Note that the subspace $D_{c}^{1}(\mathbb{T})=\left\{g \in D^{1}(\mathbb{T}): g^{\prime} \geq\right.$ $c$ ) is complete with respect to $d_{1}$ for every $c>0$. If $g \in D^{1}(\mathbb{T})$, let $x_{h, g}=$ $\operatorname{Fr}\left(g^{h}(x)\right)$ for $h \in \mathbb{Z}, x \in[0,1$ [. Moreover, when $x \in[0,1$ [, we call $g$-orbit of $x$ the set $\left\{x_{h, g}: h \in \mathbb{N}\right\}$ and $(n, g)$-orbit of $x$ the set $\left\{x_{h, g}: 0 \leq h \leq n\right\}$ for every $n \in \mathbb{N}$. 
By the discussion in Introduction, in order to construct the counterexample, it suffices to find $\bar{f}$ satisfying the following

There exist two sequences $t_{n}, t_{n}^{\prime}$ tending to $+\infty$ such that

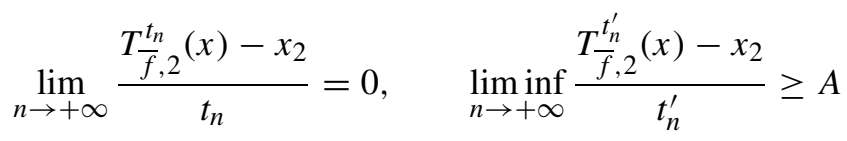

for almost all $x \in[0,1]^{2}$, where $A$ is a suitable positive constant.

It is possible to express (3.1) in terms of the pair $(\bar{g}, \bar{\Phi})$, related to $\bar{f}$ as in the previous section. Namely, as I will prove in details in Section 6, (3.1) is related to the following condition:

There exist two sequences $m_{n}, w_{n}$ of naturals tending to $+\infty$ such that

$$
\lim _{n \rightarrow+\infty} \frac{1}{m_{n}} \sum_{h=0}^{m_{n}-1} \frac{1}{\bar{\Phi}\left(\bar{g}^{h}(x)\right)}=+\infty, \quad \limsup _{n \rightarrow+\infty} \frac{1}{w_{n}} \sum_{h=0}^{w_{n}-1} \frac{1}{\bar{\Phi}\left(\bar{g}^{h}(x)\right)} \leq B
$$

for almost all $x \in[0,1]$, where $B$ is a suitable positive constant.

The relation between (3.1) and (3.2) is related to the fact that the quantity $\sum_{i=0}^{m-1} \frac{1}{\bar{\Phi}\left(\bar{g}^{i}(x)\right)}$ is comparable to the time necessary for a point $(x, 0)$, under the equation $E_{\bar{f}}$, to reach a point of the form $(u, m)$. In view of the considerations in the previous section, the function $\bar{g}$ should have an irrational Liouville rotation number $\bar{\rho}$, and $\bar{\Phi}$ should amount to 0 at 0 and should be positive on ]0, 1[. In Section 6, I will prove in fact a variant of (3.2) that is Theorem 6.1, which is more complicated than (3.2), but has the advantage that it implies (3.1) in a more direct way. I will define $(\bar{g}, \bar{\Phi})$ as the limit of a sequence $\left(g_{n}, \phi_{n}\right)$ with $\rho\left(g_{n}\right) \in \mathbb{Q}$, defined recursively. This is convenient since in view of Lemma 2.1, the maps having a rational rotation number can be better investigated. More precisely, as $\bar{\rho}$ is the limit of a sequence $\frac{p_{n}}{q_{n}}$ of rationals, I will define $g_{n}$ having $\frac{p_{n}}{q_{n}}$ as rotation number and having 0 as a periodic point, namely $g^{q_{n}}(0)=p_{n}$ (cf. Lemma 2.1). The function $\phi_{n}$ will be defined on intervals of the form $\left[\alpha_{n}, 1\right]$, with $\alpha_{n} \underset{n \rightarrow+\infty}{\longrightarrow} 0$, and extending $\phi_{n-1}$, and $\Phi$ will be the 1- periodic function extending every $\phi_{n}$. In order to define the pair $(\bar{g}, \bar{\Phi})$, we need some preparatory definitions.

Definition 3.3. We say that a continuous function $\phi:[\alpha, 1] \rightarrow \mathbb{R}$ with $0<\alpha<1$, is an $\alpha$-caliber if $\phi(1)=0$ and $0<\phi(x) \leq 1$ if $x \in[\alpha, 1[$.

If $\phi$ is an $\alpha$-caliber and $g \in D^{1}(\mathbb{T}), h \in \mathbb{N}$, we say that $x \in \mathcal{O}(g, h, \alpha)$ if $x \in\left[0,1\left[, x_{i, g} \in\right] \alpha, 1[\right.$ for $i=0, \ldots, h-1$. In such a case, we put

$$
T_{\phi}(g, h, x)=\sum_{i=0}^{h-1} \frac{1}{\phi\left(x_{i, g}\right)} .
$$

The definition of $T_{\phi}(g, h, x)$ and the following definition are suggested by formula (3.2). 
Definition 3.4. Suppose $g \in D^{1}(\mathbb{T})$, and $\phi$ is an $\alpha$-caliber. Let $\lambda, \Lambda \in \mathbb{R}$, let $w$ and $m$ be positive integers and let $J$ be the union of finitely many disjoint closed intervals. Put $\Gamma=(\lambda, \Lambda, J, w, m)$ and let $\mathcal{A}$ be the set of $\Gamma$ so obtained. We say that the pair $(g, \phi)$ is $\Gamma$ oscillating if $J \subseteq \mathcal{O}(g, m, \alpha) \cap \mathcal{O}(g, w, \alpha)$ and

$$
T_{\phi}(g, w, x) \leq \lambda w, \quad T_{\phi}(g, m, x) \geq \Lambda m, d \forall x \in J .
$$

Remark 3.5. Note that in Definition $3.4 J$ could well be empty. In such a case, clearly, $(g, \phi)$ is $\Gamma$ oscillating independently of $\Gamma \in \mathcal{A}$.

Proposition 3.6. If $(g, \phi)$ is $(\lambda, \Lambda, J, w, m)$ oscillating, then there exists $\varepsilon=$ $\varepsilon(g, \phi)>0$ such that if $g_{1} \in D^{1}(\mathbb{T}), d_{1}\left(g, g_{1}\right) \leq \varepsilon$, then $\left(g_{1}, \phi\right)$ is $(\lambda+1, \Lambda-$ $1, J, w, m)$ oscillating.

In such a case, if $d_{1}\left(g, g_{1}\right) \leq \varepsilon$ and $\phi_{1}$ is an $\alpha_{1}$-caliber extending $\phi$, we say that $\left(g_{1}, \phi_{1}\right)$ is close to $(g, \phi)$. Now, the idea of the construction consists in taking a sequence $\left(g_{n}, \phi_{n}\right)\left(\lambda_{n}, \Lambda_{n}, J_{n}, w_{n}, m_{n}\right)$ oscillating, $\phi_{n} \alpha_{n}$-caliber, each close to the previous ones and such that $\alpha_{n}$ tends to 0 as $n$ tends to infinity. If $g_{n}$ is a Cauchy sequence in $D_{c}^{1}(\mathbb{T})$ for some $c>0$, and the function on $\left.] 0,1\right]$ extending each $\phi_{n}$ can be 1-periodically extended on $\mathbb{R}$ to a $C^{\infty}$ function $\bar{\Phi}$, setting $\bar{g}=\lim g_{n}$, we then have

$$
\sum_{h=0}^{m_{n}-1} \frac{1}{\bar{\Phi}\left(\bar{g}^{h}(x)\right)} \geq\left(\Lambda_{n}-1\right) m_{n}, \quad \sum_{h=0}^{w_{n}-1} \frac{1}{\bar{\Phi}\left(\bar{g}^{h}(x)\right)} \leq\left(\lambda_{n}+1\right) w_{n},
$$

for $x \in J_{n}$. Suppose now we can choose $\Lambda_{n} \rightarrow+\infty, \lambda_{n}$ bounded, $\mu\left(J_{n}\right)>1-\frac{B}{2^{n}}$, $m_{n} \rightarrow+\infty, w_{n} \rightarrow+\infty$. Putting $\widetilde{J}=\bigcup_{k \in \mathbb{N}} \bigcap_{n \geq k} J_{n}$, we have $\mu(\widetilde{J})=1$. Therefore, if we construct $\bar{f}$ related to $\bar{g}$ and $\bar{\Phi}$ as above, we can expect that for all points in a trajectory passing through $\widetilde{J} \times 0$, possibly translated by a point in $\mathbb{Z}^{2}$, hence for almost all $x \in[0,1]^{2},(3.1)$ holds.

In conclusion, in order to prove (3.1) (or (3.2)), one can proceed in the following way: first we construct a particular pair $(g, \phi)$. Then, we have to find a sequence of $\left(g_{n}, \phi_{n}\right)$, inductively. In order to avoid problems in extending periodically on $\mathbb{R}$, we require that the derivatives of $\phi$ of every order tend to 0 at 1 , and the derivatives get smaller and smaller in the new intervals $\left[\alpha_{n+1}, \alpha_{n}[\right.$.

The natural tool for obtaining such a result would be a result stating that for every oscillating pair $(g, \phi)$ and for every $\varepsilon>0$ and for every $\Lambda^{\prime}, \lambda^{\prime}, \delta^{\prime}$ we can find a pair $(G, \Phi)\left(\Lambda^{\prime}, \lambda^{\prime}, J^{\prime}, w^{\prime}, m^{\prime}\right)$ oscillating close to $(g, \phi)$ such that $d(g, G)<\varepsilon$, with $w^{\prime}$ and $m^{\prime}$ sufficiently large, and $\mu\left(J^{\prime}\right)>1-\delta^{\prime}$. In such a case I will say that $(G, \Phi)$ derives from $(g, \phi)$. However, even restricting $\delta^{\prime}, w^{\prime}, m^{\prime}$, it does not seem simple to prove such a result. So, I will require some technical additional properties on a pair in order to complete the inductive step. In other words, I will prove the inductive step in the sense that, if we have a regular pair then we can find a new regular pair deriving from it, where by regular I mean having some specific properties. In the next two sections, I will introduce the regularity property and state the recursive lemma. 


\section{Functions of $(m, q, l, p)$ type}

In order to have a function $\bar{g} \in D^{1}(\mathbb{T})$ as above, we have to require that its rotation number is irrational, and is approximated very quickly by rational numbers. So, I will construct a sequence of functions $g_{n}$, whose rotation numbers differ by a quantity which tends to 0 very rapidly. This clarifies the reason of the following definition. In fact, given $g_{n}$ with rotation number $\frac{p_{n}}{q_{n}}$, I will construct $g_{n+1}$ with rotation number $\frac{p_{n+1}}{q_{n+1}}$ such that $\frac{p_{n+1}}{q_{n+1}}-\frac{p_{n}}{q_{n}}=\frac{1}{q_{n} q_{n+1}}$ and $q_{n+1}$ sufficiently large. In other words, I use the standard way, used for example in continued fractions, of constructing a sequence $\frac{p_{n}}{q_{n}}$ tending to an irrational (Liouville) number.

Definition 4.1. Given a function $f \in D^{1}(\mathbb{T})$, we say that $f$ is of $(m, q, l, p)$ type if $f^{q}(0)=p$ and

$$
m, q, l, p \in \mathbb{N} \backslash\{0\}, q>m+1, m p=l q+1 .
$$

Note that in such a case, the rotation number of $f$ is $\frac{p}{q}$. Moreover, it is easy to see that, once $f$ is fixed, then $m, q, l$ and $p$ are unique. The functions of $(m, q, l, p)$ type have a special importance for the following considerations, so we will investigate them in detail in this section. Note also that, if (4.1) holds, then the function $x \mapsto x+\frac{p}{q}$ is of $(m, q, l, p)$ type, and for this function $f$, $0_{h+m, f}=0_{h, f}+\frac{1}{q}$ (unless $0_{h+m, f}=0$ ), so that $0_{h+m, f}$ is the "successor" of $0_{h, f}$ (if different from 0). In view of Lemma 2.2 this happens for any function of $(m, q, l, p)$ type. Thus, the following definition of $U_{h, f}$ is, in some sense, natural. Define $\hat{a}$ for $a \in[0,1]$ as $\hat{a}=\left\{\begin{array}{l}a \text { if } a \neq 0 \\ 1 \text { if } a=0\end{array}\right.$. Put $U_{h, f}=\left[0_{h, f}, 0_{h+m, \hat{f}}[\right.$, $\mu_{h, f}=\mu\left(U_{h, f}\right)=0_{h+m, \hat{f}-0_{h, f} .}$

Lemma 4.2. Let $f$ be of $(m, q, l, p)$ type. Then $\varnothing \subset U_{h, f} \subset[0,1[$. Moreover,

i) $0<0_{m, f}<\ldots<0_{(q-1) m, f}<1=0_{q m, \hat{f}}$.

ii) If $h \equiv h^{\prime} \bmod q$, then $0_{h, f}=0_{h^{\prime}, f}$ and $U_{h, f}=U_{h^{\prime}, f}$.

iii) If $h \not \equiv h^{\prime} \bmod q$, then $0_{h, f} \neq 0_{h^{\prime}, f}$ and $U_{h, f} \cap U_{h^{\prime}, f}=\varnothing$.

iv) For every $r \in \mathbb{Z}$ the sets $U_{h, f}, h=r, \ldots, r+q-1$, are mutually disjoint and their union amounts to [0,1[. The same holds for the sets $U_{m h, f}, h=$ $0, \ldots, q-1$.

v) We have $0_{h, f} \leq 0_{q-m, f}$. Also, if $0_{h, f}>0$, in particular if $0<h<q$, then $0_{h, f} \geq 0_{m, f}$.

vi) If $h$ and $r$ are natural numbers, $r>0$, and $h+r m<q$, then $0_{h, f}<0_{h+r m, f}$.

vii) Let $h, k \in \mathbb{Z}$. Then $f^{k}$ maps $U_{h, f}$ to $\left[f^{k}\left(0_{h, f}\right)\right]+U_{h+k, f}$. More precisely, $x_{k, f}=f^{k}(x)-\left[f^{k}\left(0_{h, f}\right)\right] \in U_{h+k, f}$ for every $x \in U_{h, f}$, hence $x_{k, f}-x_{k, f}^{\prime}=$ $f^{k}(x)-f^{k}\left(x^{\prime}\right)$ if $x, x^{\prime} \in U_{h, f}$. 
Proof. The proof is a not very complicated consequence of Lemma 2.2. The details are left to the reader.

Remark 4.3. Suppose $f$ is of $(m, q, l, p)$ type. Then, in general, $f^{k}\left(0_{h, f}\right)=$ $f^{h+k}(0)-\left[f^{h}(0)\right]$ for every $h, k \in \mathbb{Z}$. As particular cases, we easily see that $f^{q}\left(0_{h, f}\right)=p+0_{h, f}$ for each $h \in \mathbb{Z}$. Moreover, $f^{m}\left(0_{h, f}\right)=0_{h+m, f}+l$ provided $0 \leq h<q-m$. Indeed, on one hand, $x+l<f^{m}(x)<x+l+1$ for every $x \in \mathbb{R}$, as if not, either $\rho(f) \leq \frac{l}{m}<\frac{p}{q}$ or $\rho(f) \geq \frac{l+1}{m}>\frac{p}{q}$, on the other, $0_{h, f}<0_{h+m, f}<$ $0_{h, f}+1$. We deduce from these considerations that $f^{j q}\left(0_{h, f}\right)=j p+0_{h, f}$ for every $j \in \mathbb{Z}$, and $f^{j m}\left(0_{h, f}\right)=0_{h+j m, f}+j l$ if $j \in \mathbb{N}$ and $0 \leq h<q-j m$.

When $f$ is of class $C^{2}$, we can control rather precisely the measure of $f^{h}(I)$ when $I$ is an interval. I here recall a standard lemma in this context, used for example for proving the Denjoy Theorem.

Lemma 4.4. Let $f \in D^{1}(\mathbb{T})$, of class $C^{2}$, and let $V=V_{f}=\operatorname{Var}\left(\log \circ f^{\prime}\right)=$ $\int_{0}^{1}\left|\frac{f^{\prime \prime}(x)}{f^{\prime}(x)}\right| d x$. Let $U$ be an interval in $\left[0,1\left[\right.\right.$, such that the sets $f^{i}(U), i=0, \ldots, h-$ 1 , are mutually disjoint on the circle, that is, $x-x^{\prime} \notin \mathbb{Z}$ if $x \in f^{i}(U), x^{\prime} \in f^{i^{\prime}}(U)$, with $i, i^{\prime}=0,1, \ldots, h-1, i \neq i^{\prime}$. Then, $e^{-V} \leq \frac{\left(f^{h}\right)^{\prime}(x)}{\left(f^{h}\right)^{\prime}\left(x^{\prime}\right)} \leq e^{V}$ for every $x, x^{\prime} \in U$.

Proof. See for example [6, Lemma 12.1.3.] Note that, although in the statement in [6], it is required that $f^{i}(U)$ are mutually disjoint for $i=0, \ldots, h$, in fact the proof there only requires this for $i=0, \ldots, h-1$.

Lemma 4.5. If $f$ is of $(m, q, l, p)$ type and of class $C^{2}$, and $h \in \mathbb{Z}$, and $x, x^{\prime} \in$ $U_{1, f}, x<x^{\prime}$, and $|h| \leq q$, then setting $V=V_{f}$, we have

i) $\frac{\mu_{h+1, f}}{\mu_{1, f}} e^{-V}\left(x^{\prime}-x\right) \leq x_{h, f}^{\prime}-x_{h, f} \leq \frac{\mu_{h+1, f}}{\mu_{1, f}} e^{V}\left(x^{\prime}-x\right)$,

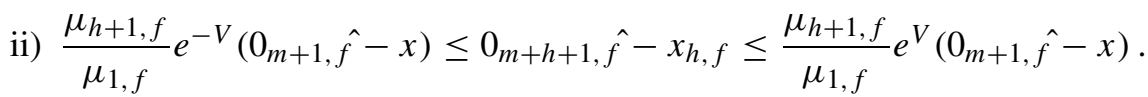

Proof. i) Suppose for example $h$ positive. Then we are in the hypothesis of Lemma 4.4, with $U=U_{1, f}$, by Lemma 4.2iv. The conclusion follows noting that both $\frac{x_{h, f}^{\prime}-x_{h, f}}{x^{\prime}-x}$ and $\frac{\mu_{h+1, f}}{\mu_{1, f}}$ amounts to $\left(f^{h}\right)^{\prime}$ at some point in $U$. If $h$ is negative, the same argument works with $U=U_{h+1, f}$. ii) This is obtained by i) taking the limit for $x^{\prime}$ tending to $0_{m+1, \hat{f}}$.

Corollary 4.6. If $f$ is of class $C^{2}$ and of $(m, q, l, p)$ type, then $e^{-V_{f}} \leq\left(f^{q}\right)^{\prime} \leq$ $e^{V_{f}}$.

Proof. For every $h \in \mathbb{Z}$ we have $\int_{U_{h, f}}\left(f^{q}\right)^{\prime}=\left(p+0_{h+m, f}\right)-\left(p+0_{h, f}\right)=$ $\mu\left(U_{h, f}\right)$, hence there exists $v \in U_{h, f}$ such that $\left(f^{q}\right)^{\prime}(v)=1$. Since every $x \in \mathbb{R}$ has the form $n+v$ for some $n \in \mathbb{Z}, v \in U_{h, f}, h \in \mathbb{Z}$, we get the desired inequalities using Lemma 4.4 again. 


\section{The recursive lemma}

I am now going to define the regularity property of an oscillating pair. To this aim, I will start by defining some special $C^{\infty}$ functions. Then, I will define more and more restricted classes of functions. Let $\hat{\psi}$ be a function of class $C^{\infty}$ from $\mathbb{R}$ to $\mathbb{R}$ such that

$$
\hat{\psi}(x)\left\{\begin{array}{l}
=0 \text { if } \quad x \geq 1 \\
=1 \text { if } \quad x \leq 0 \\
\in] 0,1[\text { if } \quad 0<x<1 .
\end{array}\right.
$$

Note that $\left|\hat{\psi}^{(j)}\right| \leq L_{j}$ for each $j \in \mathbb{N}$ and for suitable positive $L_{j}$ (which of course depend on $\hat{\psi})$ with $L_{0}=1$. For $a \leq b, u>0$, let $\hat{\psi}_{a, b, u}(x)=\hat{\psi}\left(\frac{x-b}{u}\right) \hat{\psi}\left(\frac{a-x}{u}\right)$ and let $\psi_{a, b, u}$ be the 1-periodic extension on $\mathbb{R}$ of the restriction of $\hat{\psi}_{a, b, u}$ on [0,1[. If $u<\min \{a, 1-b\}$ then $\psi_{a, b, u}$ is $C^{\infty}$.

Lemma 5.1. $0 \leq \hat{\psi}_{a, b, u}(x) \leq 1$, and

$$
\hat{\psi}_{a, b, u}(x) \begin{cases}=0 & \text { if } x \in[u+b,+\infty[\cup]-\infty, a-u] \\ =1 & \text { if } x \in[a, b] .\end{cases}
$$

Moreover, there exists $L_{j}^{\prime}>0$ such that $\left|\hat{\psi}_{a, b, u}^{(j)}\right| \leq \frac{L_{j}^{\prime}}{u^{j}}$ if $u<1, j=0,1,2,3, \ldots$

As told in beginning of previous section, given a function $f$ of $(m, q, l, p)$ type, we are now interested in finding a function slightly bigger than $f$, with strictly bigger rotation number. Related to this is the following.

Lemma 5.2. The map $f \mapsto \rho(f)$ is continuous with respect to the uniform convergence and increasing.

Proof. Cf. for example [6, Proposition 11.1.6 and Proposition 11.1.8].

However, rather surprisingly, in general, the map $f \mapsto \rho(f)$ is not strictly increasing, in other words there exist functions $f$ with rational rotation numbers such that $\rho(g)=\rho(f)$ for every $g \in D^{1}(\mathbb{T})$ with $f<g<f+\varepsilon$ for sufficiently small positive $\varepsilon$. This is a well known property of the rotation number ( $c f$. for example [6, Proposition 11.1.10]). The reason is that if $f^{q}-I d-p$ attains both positive and negative values, then, in view of Lemma 2.1 , every sufficiently small perturbation of $f$ has rotation number equal to $\frac{p}{q}$. This does not happen if $f^{q}(x) \geq$ $x+p$ for all $x$. In [2, Definition 1 in Chapter 3, Section 5], a function $g$ is called $(p, q)$-stable forward if $g^{q}(x) \geq x+p$ for all $x$ and the equality holds for at least one $x$. Here, we need a variant of such a definition.

Definition 5.3. We say that $g$ of $(m, q, l, p)$ type is $((m, q, l, p))$ sf-stable if

i) $g^{q}(x) \geq x+p d \forall x \in \mathbb{R}$,

ii) $\exists \bar{z}_{1} \in \operatorname{Int}\left(U_{1, g}\right)$ such that $g^{q}\left(\bar{z}_{1}\right)=\bar{z}_{1}+p$. 
Here, sf stands for strongly forward and Int denotes the interior of a set. For functions of ( $m, q, l, p)$ type, the equality necessarily holds for 0 (and its orbit). In ii) I require that the equality holds for some $x$ not in the orbit of 0 . I will construct $G$ derived by $g$ in several steps. At the first step, I will construct a function $\hat{g}$, summing a very small positive function $s$ to $g$. Thus, i) assures that $\rho(\hat{g})>\rho(g)$. I will now introduce the definition of a "regular" sf-stable function, and in such a class of functions I will construct $(G, \Phi)$ derived by $(g, \phi)$ (Lemma 5.5). I will now explain the idea of such a definition. However, the actual construction of $G$ could differ in some details from the ideas hinted below.

As the function $G$ will turn out to be a very small perturbation of $\hat{g}$, we will implicitly assume that $G$ has similar properties to $\hat{g}$. We want that $(G, \Phi)$ is $\left(\lambda, \Lambda^{\prime}, J^{\prime}, w^{\prime}, q\right)$ oscillating for arbitrary $\Lambda^{\prime}$ and suitable $J^{\prime}, w^{\prime}, J^{\prime}$ filling [0,1[ apart from a set of small measure. Let $\bar{I}$ be a compact interval contained in $\left[0_{1, g}, \bar{z}_{1}[\right.$, and let $x \in \bar{I}$. On one hand, if we require that $\Phi$ is positive but very small near 0 , as the $(q-1, G)$-orbit of $x$ contains the point $x_{q-1, G}$, which is very close to 0 , we have $T_{\Phi}(G, q, x) \geq \Lambda^{\prime} q$; on the other, if we could have $s\left(\bar{z}_{1}\right)=0$, then, thanks to ii), the $\hat{g}^{q}$-orbit of $\bar{z}_{1}$ would amount to $\bar{z}_{1}$, hence, using also i), $\operatorname{Fr}\left(\hat{g}^{h q}(\bar{I})\right)$ would tend to $\bar{z}_{1}$ as $h$ tends to infinity.

Thus, if we require that $s\left(\bar{z}_{1}\right)$ is positive but small in comparison to the values attained by $s$ far from $\bar{z}_{1}$, then $\operatorname{Fr}\left(\hat{g}^{h q}(\bar{I})\right)$ approaches $\bar{z}_{1}$, for $h$ sufficiently, but not too, large. It follows that, if we require $T_{\phi}\left(g, q, \bar{z}_{1}\right)<\lambda q$, then $T_{\Phi}(G, h q, x)<$ $\lambda h q$ for suitably large $h$, independent of $x \in \bar{I}$, and we can take $w^{\prime}=h q$. Of course, the same properties hold for $x$ in the set $J^{\prime}$ formed by the union of the $(q-1, G)$-orbit of the points in $\bar{I}$. In Definition 5.4, $\left[0_{1, g}, \alpha_{1-q, g}\right]$ stands for $\bar{I}$, so that $J^{\prime}$ is strictly related to the set in (5.4).

Definition 5.4. Suppose $g$ is $(m, q, l, p)$ sf-stable, $\phi$ is a $C^{\infty} \alpha$-caliber. We say that the $(\lambda, \Lambda, J, w, m)$ oscillating pair $(g, \phi)$ is $(\eta, \delta)$-regular if $q>p, \eta \in] 0,1]$, $\delta \in] 0,1\left[, \lambda \geq 1, \Lambda \geq 1\right.$ and $\alpha \in \operatorname{Int}\left(U_{0, g}\right)$ and

$$
\begin{gathered}
\alpha<\left(\bar{z}_{1}\right)_{-1, g}, \\
\phi=\eta \text { on }\left[\alpha, 0_{m, g}\right], \\
T_{\phi}\left(g, q, \bar{z}_{1}\right)<\lambda q, \\
\mu\left(\bigcup_{h=1}^{q}\left[0_{h, g}, \alpha_{h-q, g}\right]\right)>1-\delta .
\end{gathered}
$$

Lemma 5.5. Suppose $g$ is $(m, q, l, p)$ sf-stable and $C^{\infty}, \frac{1}{2}<g^{\prime}<\frac{3}{2}, \phi C^{\infty} \alpha$ caliber, $(g, \phi)(\lambda, \Lambda, J, w, m)$ oscillating, $(\eta, \delta)$-regular. Then for each

$$
\left.\varepsilon>0, \quad \delta_{1}^{\prime}>\delta, \quad \delta^{\prime} \in\right] 0,1\left[, \Lambda^{\prime} \geq 1,\right.
$$

there exist

$$
\left.\bar{\eta}>0, q^{\prime}, w^{\prime}, p^{\prime}, J^{\prime}, \alpha^{\prime}, G, r, \Phi_{\eta^{\prime}} \forall \eta^{\prime} \in\right] 0, \bar{\eta}[
$$


so that

i) $q^{\prime}>w^{\prime}>q$,

ii) $G$ is $\left(q, q^{\prime}, p, p^{\prime}\right)$ sf-stable, of class $C^{\infty}$, and $\frac{1}{2}<G^{\prime}<\frac{3}{2}$,

iii) $\Phi_{\eta^{\prime}}$ is a $C^{\infty} \alpha^{\prime}$-caliber, $\Phi_{\eta^{\prime}}=\phi$ on $[\alpha, 1]$.

iv) $0<\alpha^{\prime}<\frac{\alpha}{2}$,

v) $d_{1}(g, G)<\varepsilon$

vi) $\left(G, \Phi_{\eta^{\prime}}\right)$ is $\left(\lambda, \Lambda^{\prime}, J^{\prime}, w^{\prime}, q\right)$ oscillating $\left(\eta^{\prime}, \delta^{\prime}\right)$-regular,

vii) $\mu\left(J^{\prime}\right)>1-\delta_{1}^{\prime}$,

viii) We have $\left|\Phi_{\eta^{\prime}}^{(h)}\right| \leq L_{h} \eta r^{h}$ on $\left.] \alpha^{\prime}, \alpha\right]$.

\section{Construction of the counterexample}

In this section I construct the example using Lemma 5.5. The proof of Lemma 5.5 will be given later.

Theorem 6.1. For some $\lambda>1$ and for every sequence $\Lambda_{n} \geq 0, n \geq 1$, there exist $\bar{g} \in D^{1}(\mathbb{T})$, a function $\bar{\Phi}: \mathbb{R} \rightarrow \mathbb{R}$, a sequence $J(k)$ of measurable subsets of $\left[0,1\left[\right.\right.$, and two sequences $m_{n}, w_{n}$ of positive integers tending to infinity such that

i) $\frac{1}{2} \leq \bar{g}^{\prime} \leq \frac{3}{2}$

ii) $\bar{\Phi}$ is of class $C^{\infty}$ and 1-periodic, with values in $[0,1]$,

iii) $\bar{\Phi}(x)=0 \Longleftrightarrow x \in \mathbb{Z}$,

iv) $\mu([0,1[\backslash J(k)) \underset{k \rightarrow \infty}{\longrightarrow} 0$,

v) $\bar{g}^{h}(x) \notin \mathbb{Z}$ for every $x \in J(k)$ and $h \in \mathbb{N}$,

vi) for all $x \in J(k)$

$$
\sum_{h=0}^{m_{n}-1} \frac{1}{\bar{\Phi}\left(\bar{g}^{h}(x)\right)} \geq \Lambda_{n} m_{n}, \quad \sum_{h=0}^{w_{n}-1} \frac{1}{\bar{\Phi}\left(\bar{g}^{h}(x)\right)} \leq \lambda w_{n}, \quad \forall n \geq k .
$$

Proof. I first construct $(g, \phi)$ satisfying Lemma 5.5, such that $\phi^{(h)}(x) \underset{x \rightarrow 1}{\longrightarrow} 0$ for each $h \in \mathbb{N}$. It suffices to take

$$
g(x)=x+\frac{p}{q}, \quad m, q, l, p \in \mathbb{N} \backslash\{0\}, m p=l q+1, q>\max \{p, m+1\},
$$

and $\phi(x)=\eta \hat{\psi}\left(\frac{x-0_{m, g}}{1-0_{m, g}}\right)$. If we take $\alpha$ and $\bar{z}_{1}$ so that (5.1) holds, then $(g, \phi)$ satisfies the hypothesis of Lemma 5.5 for suitable $\delta$ and $J=\varnothing$. Thus, taking also into account Remark 3.5 , we can find inductively a sequence $\left(g_{n}, \phi_{n}\right)$, with $\left(g_{0}, \phi_{0}\right)=(g, \phi)$, such that, 
a) for $n \geq 1, g_{n}$ is $\left(m_{n}, q_{n}, l_{n}, p_{n}\right)$ sf-stable in $D_{\frac{1}{2}}^{1}(\mathbb{T})$,

b) $\phi_{n}$ is a $C^{\infty} \alpha_{n}$-caliber,

c) $\left(g_{n}, \phi_{n}\right)$ is $\left(\lambda-1, \Lambda_{n}+1, J_{n}, w_{n}, m_{n}\right)$ oscillating, $\left(\eta_{n}, \frac{1}{2^{n}}\right)$-regular,

d) $\mu\left(J_{n+1}\right)>1-\frac{2}{2^{n}}$,

e) $\alpha_{n} \underset{n \rightarrow+\infty}{\longrightarrow} 0, \eta_{n} \underset{n \rightarrow+\infty}{\longrightarrow} 0$,

f) $m_{n}<w_{n}<m_{n+1}, m_{n+1}=q_{n}$,

g) $d_{1}\left(g_{n}, g_{n+1}\right)<\frac{\min \left\{1, \varepsilon\left(g_{h}, \phi_{h}\right), h=1,2, \ldots, n\right\}}{2^{n}}$

where $\varepsilon\left(g_{h}, \phi_{h}\right)$ is as in Prop. 3.6, so that $g_{n}$ is a Cauchy sequence in $D_{\frac{1}{2}}^{1}(\mathbb{T})$. Let $\bar{g}$ be its limit. We have $d_{1}\left(g_{n}, \bar{g}\right) \leq \varepsilon\left(g_{n}, \phi_{n}\right)$ for each $n \geq 1$. Moreover, we can take $\eta_{n}$ so that

$$
L_{h} \eta_{n} r_{n}^{h} \underset{n \rightarrow+\infty}{\longrightarrow} 0
$$

for each natural $h$, where $r_{n}$ corresponds to $r$ in Lemma 5.5 viii, with $\phi_{n}$ in place of $\Phi_{\eta^{\prime}}$. Hence, we can find $\Phi$ extending every $\phi_{n}$ 1-periodic, and having the derivatives of every order at 0 equal to 0 . Using Prop. 3.6, it easily follows that $(\bar{g}, \bar{\Phi})$ satisfies the required properties with $J(k)=\bigcap_{n=k}^{+\infty} J_{n}$.

Corollary 6.2. There exist two sequences $m_{n}, w_{n}$ of naturals tending to $+\infty$ and $B>0$ such that (3.2) holds a.e. in $[0,1]$.

Once we have $(\bar{G}, \bar{\Phi})$ as in Theorem 6.1 , we can construct our example in a rather standard way. I here sketch a possible construction. I have given the statement of Corollary 6.2, since it is more understandable than Theorem 6.1. However, it is simpler to deduce our example directly from Theorem 6.1. Choose $\Lambda_{n}>0$ such that $\Lambda_{n} \underset{n \rightarrow+\infty}{\longrightarrow}+\infty$. Let $\beta_{1}(y)=\hat{\psi}(1-y)$. We can and do assume $\beta_{1}^{\prime}>0$ on ]0, $1\left[\right.$. Let $\beta_{2}=\psi_{\frac{1}{4}, \frac{3}{4}, \frac{1}{8}}$,

$$
U(x, y)=x+\beta_{1}(F r(y))(\bar{g}(x)-x), V(x, y)=(U(x, y), y)
$$

on $\mathbb{R}^{2}$. We see that $V$ is of the form $\mathrm{Id}+\mathbb{Z}^{2}$-periodic. Moreover, it is a bijection from $\mathbb{R}^{2}$ into itself, and is a $C^{1}$ diffeomorphism from $\mathbb{R}^{2} \backslash(\mathbb{R} \times \mathbb{Z})$ onto $\mathbb{R}^{2} \backslash(\mathbb{R} \times \mathbb{Z})$. As $\frac{1}{2} \leq \frac{\partial U}{\partial x}(x, y) \leq \frac{3}{2}$ on $\mathbb{R}^{2}$, we see that $V^{-1}$ has bounded partial derivatives on $\mathbb{R}^{2} \backslash(\mathbb{R} \times \mathbb{Z})$. Let now $\bar{\Gamma}: \mathbb{R}^{2} \rightarrow \mathbb{R}^{2}$ be defined by

$$
\bar{\Gamma}(x, y)=\left(\beta_{1}^{\prime}(F r(y)) \cdot(\bar{g}-I d)\left(V_{1}^{-1}(x, y)\right), 1\right),
$$

and let $\bar{f}$ be defined by

$$
\bar{f}=\gamma \bar{\Gamma}, \quad \gamma(x, y)=\beta_{2}(y) \bar{\Phi}\left(V_{1}^{-1}(x, y)\right)+1-\beta_{2}(y) .
$$


We have that $\bar{f}: \mathbb{R}^{2} \rightarrow \mathbb{R}^{2}$ is $C^{1}$ and $\mathbb{Z}^{2}$ periodic. Put

$$
\widehat{J}=\bigcup_{h \in \mathbb{Z}}\left(\left(\left[0,1\left[\backslash \bigcup_{k=1}^{+\infty} J(k)\right)+h\right), \quad t_{n}=\frac{1}{2} \Lambda_{n} m_{n}>0, \quad t_{n}^{\prime}=\lambda w_{n}>0 .\right.\right.
$$

Let $\widehat{J}^{\prime}=V(\widehat{J} \times \mathbb{R})$. It is easy to see that $\mu\left(\widehat{J}^{\prime}\right)=0$. Moreover, after some calculations we get

$$
\limsup _{n \rightarrow+\infty} \frac{T_{\bar{f}, 2}^{t_{n}}(x, y)-y}{t_{n}} \leq \limsup _{n \rightarrow+\infty} \frac{2}{\Lambda_{n}}=0, \quad \liminf _{n \rightarrow+\infty} \frac{T_{\bar{f}, 2}^{t_{n}^{\prime}}(x, y)-y}{t_{n}^{\prime}} \geq \frac{1}{\lambda}
$$

for each $(x, y) \in\left[0,1\left[^{2} \backslash \widehat{J}^{\prime}\right.\right.$. As a consequence, (2.1) and (2.2) hold.

Remark 6.3. We can in fact take $\bar{f}$ of class $C^{\infty}$, as we can choose $G$ to be arbitrarily close to $g$ in the $C^{\infty}$ metric in Lemma 5.5. To this aim it suffices to take $\varsigma$ sufficiently small in the construction of $\tilde{g}$ in Section 7, and $\hat{\varepsilon}$ sufficiently small in the construction of $G$ in Section 8.

\section{Construction of a function of $\left(q, q^{\prime}, p, p^{\prime}\right)$ type}

The rest of this paper is devoted to prove Lemma 5.5. So, from now on, we will assume that $g$ is as in Lemma 5.5. In this section I describe the first two steps in the construction of $G$, obtaining a function $\tilde{g}$ which is of $\left(q, q^{\prime}, p, p^{\prime}\right)$ type, but not necessarily sf-stable. I preliminarily introduce some constants. Let

$$
\tilde{M}=\frac{\mu\left(\bigcup_{h=1}^{q}\left[0_{h, g}, \alpha_{h-q, g}\right]\right)-(1-\delta)}{q\left(\frac{3}{2}\right)^{q-1}} .
$$

Note that, in view of (5.4), we have $\widetilde{M}>0$. In order to introduce the other constants, we have to observe that

$$
0_{1, g}<\alpha_{1-q, g}-\tilde{M}<\alpha_{1-q, g} \leq \alpha_{1, g}<\bar{z}_{1}<0_{m+1, g} .
$$

In fact, we have

$$
\begin{aligned}
\mu\left(\bigcup_{h=1}^{q}\left[0_{h, g}, \alpha_{h-q, g}\right]\right) & \leq \sum_{h=1}^{q} \alpha_{h-q, g}-0_{h, g}=\sum_{h=1}^{q}\left(\alpha_{1-q, g}\right)_{h-1, g}-\left(0_{1, g}\right)_{h-1, g} \\
& =\sum_{h=1}^{q} g^{h-1}\left(\alpha_{1-q, g}\right)-g^{h-1}\left(0_{1, g}\right) \\
& =\left(\alpha_{1-q, g}-0_{1, g}\right) \sum_{h=1}^{q}\left(g^{h-1}\right)^{\prime}\left(\xi_{h}\right) \\
& \leq\left(\alpha_{1-q, g}-0_{1, g}\right) \sum_{h=1}^{q}\left(\frac{3}{2}\right)^{h-1} \leq\left(\alpha_{1-q, g}-0_{1, g}\right) q\left(\frac{3}{2}\right)^{q-1}
\end{aligned}
$$


for suitable $\xi_{h}$, hence the first inequality holds, the second is trivial, the fourth and the fitfh follow from Definition 5.3ii, (5.1) and Lemma 4.2vii, and the third follows from Definition 5.3i (cf. Corollary 7.4iv). Now, let $\bar{z}_{2}$ and $\sigma>0$ be so that

$$
\begin{aligned}
0_{1, g}< & \alpha_{1-q, g}-\tilde{M}<\bar{z}_{2}<\alpha_{1-q, g} \\
& \leq \alpha_{1, g}<\bar{z}_{1}-2 \sigma<\bar{z}_{1}+2 \sigma<0_{m+1, g}, \\
T_{\phi}(g, q, x)< & \lambda q \quad \forall x \in \tilde{I}:=\left[\bar{z}_{1}-2 \sigma, \bar{z}_{1}+2 \sigma\right], \\
g^{q}(x)< & x+p+\frac{\sigma}{4} \quad \forall x \in \tilde{I} .
\end{aligned}
$$

Note that (7.2) holds for sufficiently small $\sigma$ by (5.3), and (7.3) holds for small $\sigma$ as $g^{q}-I d$ takes its minimum at $\bar{z}_{1}$, hence $\left(g^{q}-I d\right)^{\prime}\left(\bar{z}_{1}\right)=0$. I will use (7.1) without reference. By the definition of $\widetilde{M}$ we have

$$
\mu\left(\bigcup_{h=1}^{q}\left[0_{h, g},\left(\bar{z}_{2}\right)_{h-1, g}\right]\right)>1-\delta .
$$

Indeed,

$$
\begin{aligned}
\mu\left(\bigcup_{h=1}^{q}\left[0_{h, g},\left(\bar{z}_{2}\right)_{h-1, g}\right]\right)= & \sum_{h=1}^{q}\left(\bar{z}_{2}\right)_{h-1, g}-\left(0_{1, g}\right)_{h-1, g} \\
& >\sum_{h=1}^{q}\left(\alpha_{1-q, g}\right)_{h-1, g}-\left(0_{1, g}\right)_{h-1, g} \\
& -\sum_{h=1}^{q}\left(\alpha_{1-q, g}\right)_{h-1, g}-\left(\alpha_{1-q, g}-\tilde{M}\right)_{h-1, g} \\
= & \mu\left(\bigcup_{h=1}^{q}\left[0_{h, g}, \alpha_{h-q, g}\right]\right) \\
& -\sum_{h=1}^{q}\left(g^{h-1}\left(\alpha_{1-q, g}\right)-g^{h-1}\left(\alpha_{1-q, g}-\tilde{M}\right)\right) \\
= & \mu\left(\bigcup_{h=1}^{q}\left[0_{h, g}, \alpha_{h-q, g}\right]\right)-\tilde{M} \sum_{h=1}^{q}\left(g^{h-1}\right)^{\prime}\left(\xi_{h}\right)
\end{aligned}
$$

for suitable $\xi_{h}$, and we use, as before, the assumption $\frac{1}{2} \leq g^{\prime} \leq \frac{3}{2}$ and the definition of $\widetilde{M}$ to deduce (7.4). I will now introduce a function $\hat{g}$, obtained by $g$ summing a small positive function around $U_{1, g}$, which is even smaller around $\bar{z}_{1}$. In the following construction, we have to take $\sigma$ and $\tau$ to be small positive numbers, and $\tau$ small in comparison to $\sigma$. Let $\widetilde{\sigma}>0$ be such that

$$
\tilde{\sigma}<\min \left\{0_{1, g}-\left(\bar{z}_{1}\right)_{q-m, g},\left(\bar{z}_{1}\right)_{m, g}-0_{m+1, g}\right\} .
$$


For $\varsigma>0$ and $\tau \in[0, \varsigma]$, let $\psi_{((1))}=\psi_{0_{1, g}, 0_{m+1, g}, \tilde{\sigma}}, \psi_{((2))}=\psi_{\bar{z}_{1}, \bar{z}_{1}, \sigma}$,

$$
(\hat{g}=) \hat{g}_{\zeta, \tau}=g+\varsigma \psi_{((1))}+(\tau-\varsigma) \psi_{((2))} .
$$

Note that, in view of (7.5), $\hat{g}=g$ on $\left[0,1[\backslash]\left(\bar{z}_{1}\right)_{q-m, g},\left(\bar{z}_{1}\right)_{m, g}[\right.$.

From now on I will assume that $\varsigma$ is sufficiently small, in other words, in every statement, I will mean: there exists $\bar{\zeta}>0$ such that the statement holds if $0<\varsigma<$ $\bar{\zeta}$, independently of $\tau$.

I will explicitly say "for small $\varsigma$ " in sentences like: "given $\omega$, for small $\varsigma$ " to state that there exists $\bar{\zeta}(\omega)>0$ such that the property holds if $0<\varsigma<\bar{\zeta}(\omega)$, independently of $\tau$. We can assume that $\frac{1}{2}<\hat{g}^{\prime}<\frac{3}{2}$, hence $\hat{g} \in D^{1}(\mathbb{T})$. Also,

$$
\left|\hat{g}^{\prime \prime}(x)\right| \leq \max \left|g^{\prime \prime}\right|+1 \text {. }
$$

Now, the idea of the next lemmas consists in fixing a sufficiently small $\zeta$, and finding a suitable $\tau$ useful for our considerations.

Lemma 7.1. $\hat{g}_{5,0}^{q}\left(\bar{z}_{1}\right)=\bar{z}_{1}+p$, hence $\rho\left(\hat{g}_{5,0}\right)=\frac{p}{q}$. If $\left.\left.\tau \in\right] 0, \varsigma\right]$, then $\hat{g}_{5, \tau}^{q}>$ Id $+p$, hence $\rho\left(\hat{g}_{5, \tau}\right)>\frac{p}{q}$. Moreover, the map $\tau \mapsto \rho\left(\hat{g}_{5, \tau}\right)$ is continuous and increasing on $[0, \varsigma]$.

Proof. For each $x \in U_{1, g}$ we have $\psi_{((1))}(x)=1$, hence $\hat{g}_{5, \tau}(x) \geq g(x)+\tau$. For $x \in\left[0,1\left[\backslash U_{1, g}\right.\right.$ we have $\psi_{((2))}(x)=0$, hence $\hat{g}_{5, \tau}(x) \geq g(x)$. If $\tau>0$, as every $(q-1, g)$-orbit meets $U_{1, g}$, it follows that $\hat{g}_{5, \tau}^{q}>g^{q} \geq I d+p$ as $g$ is sf-stable. On the other hand, as $g=\hat{g}_{5,0}$ on $B:=\left(\left[0,1[\backslash]\left(\bar{z}_{1}\right)_{q-m, g},\left(\bar{z}_{1}\right)_{m, g}[) \cup\left\{\bar{z}_{1}\right\}\right.\right.$, and the $(q-1, g)$-orbit of $\bar{z}_{1}$ is contained in $B$, then $\hat{g}_{5,0}^{q}\left(\bar{z}_{1}\right)=g^{q}\left(\bar{z}_{1}\right)=\bar{z}_{1}+p$ and $\rho\left(\hat{g}_{\zeta, 0}\right)=\frac{p}{q}$. Since $\hat{g}_{\zeta, \tau}-\hat{g}_{\zeta, \tau^{\prime}}=\left(\tau-\tau^{\prime}\right) \psi_{((2))}$, the last part of the statement follows from Lemma 5.2.

Lemma 7.2. For each $\omega>0$ and for each $\varsigma>0$ there exist $p^{\prime}$ and $q^{\prime}$, and $\tau \in$ ]0, 5 [ so that

$$
\begin{gathered}
\frac{1}{q q^{\prime}}=\frac{p^{\prime}}{q^{\prime}}-\frac{p}{q}<\omega, p^{\prime}, q^{\prime} \in \mathbb{N} \backslash\{0\}, q^{\prime}>q+1, \\
\tau=\max \left\{t \in[0, \varsigma]: \rho\left(\hat{g}_{5, t}\right)=\frac{p^{\prime}}{q^{\prime}}\right\} .
\end{gathered}
$$

Proof. We have $-l q-(-m) p=1$. Put $\alpha_{n}=-l+n p, \beta_{n}=-m+n q$. Then, it suffices to take $p^{\prime}=\alpha_{n}$ and $q^{\prime}=\beta_{n}$ for sufficiently large $n$, and we get (7.7). Replacing $\omega$ by $\omega \wedge\left(\rho\left(\hat{g}_{5, \varsigma}\right)-\frac{p}{q}\right)$, then $\tau$ defined by (7.8) satisfies $0<\tau<\varsigma$. 
In the following, I will consider always $\zeta, \tau$ satisfying the previous lemma. In such a case we will say that $\tau$ is $\zeta$-regular. Note that $\hat{g}$ is not necessarily of $\left(q, q^{\prime}, p, p^{\prime}\right)$ type, as we are not guaranteed that $\hat{g}^{q^{\prime}}(0)=p^{\prime}$, so that we have to slightly modify it to obtain a function $\tilde{g}$ of $\left(q, q^{\prime}, p, p^{\prime}\right)$ type. I will now define $\tilde{g}$ and prove its main properties. Let $\hat{g}_{a}$ be the function defined by $\hat{g}_{a}(x)=\hat{g}(x+$ $a)-a$ for every $a \in \mathbb{R}$.

Lemma 7.3. There exists $\bar{a}=\bar{a}_{5, \tau} \in\left[0, \hat{g}_{5, \tau}^{q}(0)-p\right.$ [ such that $\tilde{g}=\hat{g}_{\bar{a}}$ is of $\left(q, q^{\prime}, p, p^{\prime}\right)$ type.

Proof. By Lemma $7.1 \hat{g}^{q}-I d$ has a minimum $>p$. Since $\rho(\hat{g})=\frac{p^{\prime}}{q^{\prime}}$ there exists $b \in \mathbb{R}$ such that $\hat{g}^{q^{\prime}}(b)=b+p^{\prime}$. Then every $a$ of the form $\left(\hat{g}^{q}-p\right)^{r}(b)$ satisfies $\hat{g}^{q^{\prime}}(a)=a+p^{\prime}$, so that $\hat{g}_{a}$ is of $\left(q, q^{\prime}, p, p^{\prime}\right)$ type. If we take the minimum integer $r$ such that $\left(\hat{g}^{q}-p\right)^{r}(b) \geq 0$, then $a \in\left[0, \hat{g}_{5, \tau}^{q}(0)-p[\right.$.

Note that $\tilde{g}$ in fact depends on $\varsigma, \tau$, but in order to simplify the notation, I will write simply $\tilde{g}$ and not $\tilde{g}_{\zeta, \tau}$ or similar.

Corollary 7.4. i) $\tilde{g}$ is $C^{\infty}$ and its rotation number amounts to $\frac{p^{\prime}}{q^{\prime}}<1$, thus $x<\tilde{g}(x)<x+1$ for each $x \in \mathbb{R}$. Moreover, $\tilde{g}^{q}(x)>x+p$ for each $x \in \mathbb{R}$.

ii) $\tilde{g}^{q^{\prime}}(x) \geq x+p^{\prime}$.

iii) $\frac{1}{2}<\tilde{g}^{\prime}<\frac{3}{2}$.

iv) $x_{q^{\prime}, \tilde{g}} \geq x$ for each $x \in[0,1[$.

v) For each $\omega>0$ for small $\varsigma$ we have $d_{1}(g, \tilde{g}) \vee d_{1}(\hat{g}, \tilde{g})<\omega$.

Proof. i) and iii) are trivial. If ii) is false, then the analogous statement for $\hat{g}$ is also false and we easily get a contradiction with (7.8). Prove now iv). We have $p^{\prime} \leq x+p^{\prime} \leq \tilde{g}^{q^{\prime}}(x)<\tilde{g}^{q^{\prime}}(1)=p^{\prime}+1$, hence $\left[\tilde{g}^{q^{\prime}}(x)\right]=p^{\prime}$, and we use ii). To prove v), use the definition of $\hat{g}$ and $\tilde{g}$ and note that for small $\zeta, \bar{a}_{\zeta, \tau}$ can be made as small as possible, as $g^{q}(0)=p$.

Corollary 7.5. i) For every $v>0$ and $\varsigma>0$ there exists $\tau$ $\varsigma$-regular such that $\tau \in] 0, v[$.

ii) For every $k>0$ there exists $\tau(k, \varsigma)>0$ such that for every $\tau \varsigma$-regular in ] $0, \tau(k, \varsigma)\left[\right.$ we have $q^{\prime}>k$.

Proof. This follows from Lemma 7.1 and Lemma 7.2.

Corollary 7.6. i) For every $\omega>0$ and $M$ positive integer, for small $\varsigma$ and for each $h \in \mathbb{Z}$ so that $|h| \leq M$, we have $d_{1}\left(\hat{g}^{h}, \tilde{g}^{h}\right) \vee d_{1}\left(g^{h}, \tilde{g}^{h}\right)<\omega$.

ii) Given $\omega>0, x \in\left[0,1\left[\right.\right.$, and $h \in \mathbb{Z}$ such that $x_{h, g} \neq 0$, for small $\varsigma$ we have $\left[\tilde{g}^{h}(x)\right]=\left[g^{h}(x)\right]$ and $\left|x_{h, \tilde{g}}-x_{h, g}\right|<\omega$.

Proof. i) follows from the definition of $\hat{g}$ and Lemma 3.2, and ii) follows from i). 
Corollary 7.7. For each $k>0$, for small $\varsigma$ we have $q^{\prime}>k$.

In order to prove Lemma 5.5, I will now introduce the constants $\alpha^{\prime}, \bar{z}^{\prime}$, necessary for proving that $G$ and $\Phi$ have the required properties, and other constants which will play an auxiliary role. Let

$$
V_{1}=2\left(\max \left|g^{\prime \prime}\right|+1\right), \quad V_{2}=e^{V_{1}} .
$$

Then, thanks to (7.6) and Corollary 7.4iii, we have $V_{\tilde{g}} \leq V_{1}$. Let $\alpha^{\prime}, \bar{z}^{\prime}, \hat{\varepsilon}, \bar{u}_{1}$, be such that $\alpha_{1, \tilde{g}}^{\prime}, \bar{z}^{\prime} \in \operatorname{Int}\left(U_{1, \tilde{g}}\right)$ and

$$
\begin{gathered}
\frac{9}{2} V_{2}^{2} \frac{0_{q+1, \hat{g}}-\bar{z}^{\prime}}{\mu_{1, \tilde{g}}}<\frac{0_{q+1, \hat{\tilde{g}}}-\alpha_{1, \tilde{g}}^{\prime}}{\mu_{1, \tilde{g}}}<\frac{\delta^{\prime}}{V_{2}^{2}}, \\
0<\hat{\varepsilon}<\min \left\{\frac{\varepsilon}{2\left(L_{1}^{\prime}+1\right)}, \frac{\left(\min g^{\prime}-\frac{1}{2}\right) \wedge\left(\frac{3}{2}-\max g^{\prime}\right)}{2 L_{1}^{\prime}}\right\}, \\
\bar{u}_{1}=\frac{0_{q+1, \hat{g}}-\bar{z}^{\prime}}{4 V_{2}}>0 .
\end{gathered}
$$

Recall that $L_{1}^{\prime}$ is defined in Lemma 5.1. Note that in particular $\hat{\varepsilon}<\frac{\varepsilon}{2}$. Note also that the inequality $\bar{u}_{1}>0$ follows from the definition of $\bar{z}^{\prime}$. I also stress that, although $\alpha^{\prime}$ and $\bar{z}^{\prime}$ could depend on $\varsigma$ and $\tau$, we can and do choose the ratios $\frac{0_{q+1, \hat{g}}-\bar{z}^{\prime}}{\mu_{1, \tilde{g}}}, \frac{0_{q+1, \hat{g}}-\alpha_{1, \tilde{g}}^{\prime}}{\mu_{1, \tilde{g}}}$ not depending on $\varsigma$ and on $\tau$. Note that it follows $\bar{z}^{\prime}>$ $\alpha_{1, \tilde{g}}^{\prime}$. Formula (7.9) will be used in Lemma 7.9, and (7.10) will be used in Section 8.

An important feature of $g$ is that $g^{h q}-I d$ takes its minimum at $0_{1, g}$, so that $g^{h q}$ is a good approximation of the function $x+h p$, for $x$ close to $0_{1, g}$. This is the inspiring idea of the properties described below.

Lemma 7.8. For every integer $M_{1}>0$ and $\left.\omega_{1} \in\right] 0, \frac{1}{2}[$ for small $\varsigma$

$$
\begin{gathered}
1-\omega_{1}<\left(\tilde{g}^{h q}\right)^{\prime}(x)<1+\omega_{1} \text { if } h=0, \ldots, M_{1}, x \in \bigcup_{k=0}^{M_{1}} U_{k q+1, \tilde{g}} \\
=\left[0_{1, \tilde{g}}, 0_{1+\left(M_{1}+1\right) q, \hat{g}[}[\right. \\
q^{\prime}>M_{1} q+1, \\
h p \leq \tilde{g}^{h q}(0)<\tilde{g}^{h q+1}(0)<h p+1, \quad \forall h=0, \ldots, M_{1},
\end{gathered}
$$

and the first inequality in (7.14) is strict if $h>0$. 
Proof. For every $h \in \mathbb{N}$ the function $g^{h q}-I d$ is $\geq h p$ and amounts to $h p$ at $0_{1, g}$, so that it takes its minimum at $0_{1, g}$. Therefore, $\left(g^{h q}\right)^{\prime}\left(0_{1, g}\right)=1$. We deduce

$$
\left.\left|\left(g^{h q}\right)^{\prime}(x)-1\right|<\frac{\omega_{1}}{2} \forall h=0, \ldots, M_{1}, x \in\right] 0_{1, g}-\omega_{2}, 0_{1, g}+\omega_{2}[
$$

for small $\omega_{2}>0$, and (7.13) follows from Corollary 7.7. Thus, if (7.14) is false, then either $\rho(\tilde{g}) \leq \frac{p}{q}<\frac{p^{\prime}}{q^{\prime}}$ or $\rho(\tilde{g}) \geq \frac{h p+1}{h q+1}>\frac{p^{\prime}}{q^{\prime}}$, a contradiction. As for small $\varsigma$ the previous formulas hold for $M_{1}+1$ in place of $M_{1}$ and $\omega_{2} \wedge \frac{\omega_{1}}{2}$ in place of $\omega_{1}$, in view of Corollary 7.6, we easily get for small $\varsigma$ and $h=0, \ldots, M_{1}+1$

$$
\left|0_{h q+1, \tilde{g}}-0_{1, g}\right|=\left|\tilde{g}^{h q+1}(0)-g^{h q+1}(0)\right| \leq d_{1}\left(\tilde{g}^{h q+1}, g^{h q+1}\right)<\omega_{2} .
$$

Hence, for $\left.h, k=0, \ldots, M_{1}, U_{k q+1, \tilde{g}} \subseteq\right] 0_{1, g}-\omega_{2}, 0_{1, g}+\omega_{2}[$, hence using (7.15), and the inequality $\left|\left(\tilde{g}^{h q}\right)^{\prime}(x)-\left(g^{h q}\right)^{\prime}(x)\right|<\frac{\omega_{1}}{2}$ valid for each real $x$, we get (7.12).

Lemma 7.9. If $h=0, \ldots, M_{1}$, and $M_{1}$ is as in Lemma 7.8, hence $0 \leq h q<q^{\prime}$, then

i) $0_{h q+1, \tilde{g}} \leq \alpha_{h q+1, \tilde{g}}^{\prime}<\bar{z}_{h q-q^{\prime}, \tilde{g}}^{\prime}-2 V_{2} \bar{u}_{1}<\bar{z}_{h q, \tilde{g}}^{\prime}+2 V_{2} \bar{u}_{1}<0_{h q+q+1, \hat{g} .}$

ii) $0 \leq \bar{z}_{h q, \tilde{g}}^{\prime}-\bar{z}_{h q-q^{\prime}, \tilde{g}}^{\prime} \leq 6 V_{2}\left(V_{2}-1\right) \bar{u}_{1}$.

Proof. i) The first inequality is trivial. In order to prove the second inequality, it suffices to note that in view of Lemma 7.8, Lemma 4.5 ii with $k=-q^{\prime}$, and (7.9)

$$
\begin{gathered}
\bar{z}_{h q-q^{\prime}, \tilde{g}}^{\prime}-\alpha_{h q+1, \tilde{g}}^{\prime} \geq \frac{1}{2}\left(\bar{z}_{-q^{\prime}, \tilde{g}}^{\prime}-\alpha_{1, \tilde{g}}^{\prime}\right), \\
0_{q+1, \hat{g}}-\alpha_{1, \tilde{g}}^{\prime}>\left(V_{2}+1\right)\left(0_{q+1, \hat{g}}-\bar{z}^{\prime}\right), \quad 0_{q+1, \hat{g}}-\bar{z}_{-q^{\prime}, \tilde{g}}^{\prime} \leq V_{2}\left(0_{q+1, \hat{g}}-\bar{z}^{\prime}\right) .
\end{gathered}
$$

The third inequality is an immediate consequence of Corollary 7.4iv, and the fourth follows from Lemma 7.8 and the definition of $\bar{u}_{1}$. We have proved i). To prove ii), note that

$$
\bar{z}^{\prime}-\bar{z}_{-q^{\prime}, \tilde{g}}^{\prime}=\left(0_{q+1, \hat{g}}-\bar{z}_{-q^{\prime}, \tilde{g}}^{\prime}\right)-\left(0_{q+1, \hat{g}}-\bar{z}^{\prime}\right)
$$

and use an argument like the previous one.

\section{Construction and properties of $G$}

The function $\tilde{g}$ could appear to be a good candidate for $G$, but it is not sf-stable in that it satisfies i), but not necessarily ii) in Definition 5.3. Hence, we have to make the function smaller by a carefully chosen quantity that allows it to satisfy both i) and ii) in Definition 5.3. I will define functions $G_{j, \zeta}$ by recursion on $j$, starting from $\tilde{g}$, and then I will choose particular $j$ and $\zeta$. At every step we subtract 
functions that have mutually disjoint supports, thus we finally subtract a very small quantity but in many intervals, and not a relatively big quantity in one interval, in order to keep the difference small in the distance $C^{1}$. Define $G_{j, \zeta}$ for $\zeta \in[0, \hat{\varepsilon}]$ by recursion as

$$
\left\{\begin{array}{l}
G_{0, \zeta}=\tilde{g} \\
G_{j+1, \zeta}=G_{j, \zeta}-\zeta \bar{u}_{1} \psi_{\gamma_{j}(\zeta), \gamma_{j}(\zeta), \bar{u}_{1}} \forall j \in \mathbb{N}
\end{array}\right.
$$

where $\tilde{\gamma}_{j}(\zeta)=G_{j, \zeta}^{j q-1}\left(\bar{z}^{\prime}\right)$ and $\gamma_{j}(\zeta)=\operatorname{Fr}\left(\tilde{\gamma}_{j}(\zeta)\right)$. Note that we have $G_{j+1, \zeta} \leq$ $G_{j, \zeta} \leq \tilde{g}$. Also, $G_{j, 0}=\tilde{g}$ for each $j \in \mathbb{N}$. In the next lemma I prove the main properties of $G_{j, \zeta}$.

Lemma 8.1. There exists $\bar{j}>0$ with $\bar{j} q+1<q^{\prime}$ such that for every $j=0, \ldots, \bar{j}$ we have
a) ${ }_{j} G_{j, \zeta}$ is of $\left(q, q^{\prime}, p, p^{\prime}\right)$ type and of class $C^{\infty}$,
b) $j \frac{1}{2}<G_{j, \zeta}^{\prime}<\frac{3}{2}$,
c) ${ }_{j}$ the map $\zeta \mapsto G_{j, \zeta}$ from $[0, \hat{\varepsilon}]$ to $\left(D^{1}(\mathbb{T}), d_{0}\right)$ is continuous,
d) ${ }_{j}$ the map $\zeta \mapsto G_{j, \zeta}^{j q}\left(\bar{z}^{\prime}\right)$ from $[0, \hat{\varepsilon}]$ to $\mathbb{R}$ is decreasing,
e) $j \bar{u}_{1}+0_{j q, \tilde{g}}+j p<\tilde{g}^{-1}\left(\bar{z}_{j q-q^{\prime}, \tilde{g}}^{\prime}\right)+j p \leq \tilde{\gamma}_{j}(\zeta) \leq \tilde{g}^{j q-1}\left(\bar{z}^{\prime}\right)<-\bar{u}_{1}+$ $0_{j q+q, \tilde{g}}+j p, \quad$ for $j<\bar{j}$,
f) $j$ for $j>0 G_{j, \zeta}(x)=G_{j-1, \zeta}(x)$ for each $x \in\left[0,1\left[\backslash \backslash \operatorname{Int}\left(U_{j q-q, \tilde{g}}\right)\right.\right.$,
g) $G_{\bar{j}, \hat{\varepsilon}}^{\bar{j} q}\left(\bar{z}^{\prime}\right)<\bar{z}_{\bar{j} q-q^{\prime}, \tilde{g}}^{\prime}+\bar{j} p$,
h) $G_{j, \hat{\varepsilon}}^{j q}\left(\bar{z}^{\prime}\right) \geq \bar{z}_{j q-q^{\prime}, \tilde{g}}^{\prime}+j p$ for $j<\bar{j}$,
i) ${ }_{j} d_{1}\left(G_{j, \zeta}, g\right)<\varepsilon, d_{1}\left(G_{j, \zeta}, \tilde{g}\right)<\left(1+L_{1}^{\prime}\right) \hat{\varepsilon}$,
1) $j$ For every $h, k \in \mathbb{Z}, x \in \mathbb{R}$ we have $G_{j, \zeta}^{k}\left(0_{h, \tilde{g}}\right)=\tilde{g}^{k}\left(0_{h, \tilde{g}}\right), 0_{h, \tilde{g}}=0_{h, G_{j, \zeta}}$, $\left[G_{j, \zeta}^{k}(x)\right]=\left[\tilde{g}^{k}(x)\right]$.

Proof. I will prove the lemma by recursion in the following way. For large $M_{1}$

$1^{\text {st }}$ step. a $\left.\left.\left.\left.\left.\left.\left.)_{j}, \mathrm{~b}\right)_{j}, \mathrm{c}\right)_{j}, \mathrm{~d}\right)_{j}, \mathrm{f}\right)_{j}, \mathrm{~h}\right)_{j}, \mathrm{i}\right)_{j}, \mathrm{l}\right)_{j}$ hold for $j=0$.

$2^{\text {nd }}$ step. If $0 \leq j<M_{1}$ and a $\left.\left.\left.\left.\left.\left.\left.)_{r}, \mathrm{~b}\right)_{r}, \mathrm{c}\right)_{r}, \mathrm{~d}\right)_{r}, \mathrm{f}\right)_{r}, \mathrm{~h}\right)_{r}, \mathrm{i}\right)_{r}, \mathrm{l}\right)_{r}$, hold for $r=0, \ldots, j$, then e) $)_{r}$ holds for $r=0, \ldots, j$ and a) $\left.\left.\left.\left.\left.{ }_{j+1}, \mathrm{~b}\right)_{j+1}, \mathrm{c}\right)_{j+1}, \mathrm{~d}\right)_{j+1}, \mathrm{f}\right)_{j+1}, \mathrm{i}\right)_{j+1}$, 1) $j+1$ hold.

$3^{\text {rd }}$ step. There exists $j=0, \ldots, M_{1}$ such that $\left.\mathrm{h}\right)_{j}$ does not hold. This implies $\bar{j} \leq M_{1}$.

The first step is trivial (independently of $M_{1}$ ). Fix now $M_{1}>1$ and $\left.\omega_{1} \in\right] 0, \frac{1}{2}$ [ so that

$$
\hat{\varepsilon} \sum_{h=1}^{M_{1}-1}\left(1-\omega_{1}\right)^{h}>6 V_{2}\left(V_{2}-1\right) \text {. }
$$


I now prove the second step. Let $\varsigma$ be so small that Lemma 7.8 holds. Assume the hypothesis of second step, fix $r=0, \ldots, j$ and prove e $)_{r}$. Note that, in view of (7.14), $\tilde{g}^{-1}\left(0_{r q+1, \tilde{g}}\right)=0_{r q, \tilde{g}}, \tilde{g}^{r q-1}\left(0_{q+1, \tilde{g}}\right)=0_{r q+q, \tilde{g}}+r p$. We thus have

$$
\begin{aligned}
\tilde{\gamma}_{r}(\zeta) & \geq \tilde{g}^{-1}\left(G_{r, \zeta}^{r q}\left(\bar{z}^{\prime}\right)\right) \geq \tilde{g}^{-1}\left(G_{r, \hat{\varepsilon}}^{r q}\left(\bar{z}^{\prime}\right)\right) \\
& \geq \tilde{g}^{-1}\left(\bar{z}_{r q-q^{\prime}, \tilde{g}}^{\prime}+r p\right) \geq \tilde{g}^{-1}\left(0_{r q+1, \tilde{g}}+2 V_{2} \bar{u}_{1}\right)+r p \\
& >\bar{u}_{1}+\tilde{g}^{-1}\left(0_{r q+1, \tilde{g}}\right)+r p=\bar{u}_{1}+0_{r q, \tilde{g}}+r p
\end{aligned}
$$

where I have used Lemma 7.9i and Corollary 7.4iii. On the other hand,

$$
\begin{aligned}
\tilde{\gamma}_{r}(\zeta) & =G_{r, \zeta}^{r q-1}\left(\bar{z}^{\prime}\right) \leq \tilde{g}^{r q-1}\left(\bar{z}^{\prime}\right) \\
& <\tilde{g}^{r q-1}\left(0_{q+1, \tilde{g}}-2 V_{2} \bar{u}_{1}\right)<-2 V_{2} \bar{u}_{1} \frac{1}{3}+\tilde{g}^{r q-1}\left(0_{q+1, \tilde{g}}\right) \\
& <0_{r q+q, \tilde{g}}+r p-\bar{u}_{1}
\end{aligned}
$$

where I have used Lemma 7.8, Corollary 7.4iii and the obvious inequality $2 V_{2}>3$. In conclusion, e) ${ }_{r}$ holds. As e) ${ }_{r}$ implies $\gamma_{r}(\zeta)=\tilde{\gamma}_{r}(\zeta)-r p$, we have $0<\bar{u}_{1}+$ $0_{j q, \tilde{g}}<\gamma_{j}(\zeta)<0_{j q+q, \tilde{g}}-\bar{u}_{1}<1$ and f) ${ }_{j+1}$ follows from Lemma 5.1. I now prove b) ${ }_{j+1}$. Since the sets where $G_{r+1, \zeta}-G_{r, \zeta} \neq 0$ restricted to [0, 1[, being contained in $U_{r q, \tilde{g}}$, for $r=0, \ldots, j$, are mutually disjoint by Lemma 4.2 iv as $0 \leq r<q^{\prime}$, we have

$$
\left|G_{j+1, \zeta}^{\prime}(x)-\tilde{g}^{\prime}(x)\right| \leq \max _{0 \leq r \leq j} \zeta \bar{u}_{1}\left|\psi_{\gamma_{r}(\zeta), \gamma_{r}(\zeta), \bar{u}_{1}}^{\prime}(x)\right| \leq L_{1}^{\prime} \zeta \leq L_{1}^{\prime} \hat{\varepsilon}
$$

so that b) ${ }_{j+1}$ holds by (7.10) and Corollary $7.4 \mathrm{v}$ with $\omega=L_{1}^{\prime} \hat{\varepsilon}$. By proceeding in a similar way, as clearly $\bar{u}_{1}<1$, we get

$$
\left|G_{j+1, \zeta}(x)-\tilde{g}(x)\right| \leq \zeta \bar{u}_{1} \leq \zeta \leq \hat{\varepsilon} .
$$

Hence, using Corollary $7.4 \mathrm{v}$ again we get i $)_{j+1}$. In view of e) ${ }_{j}$, we have $\gamma_{j}(\zeta)=$ $G_{j, \zeta}^{j q-1}\left(\bar{z}^{\prime}\right)-j p$, so that c) $)_{j+1}$ follows from c) ${ }_{j}$ and a simple continuity argument. We have

$$
G_{j+1, \zeta}^{k}\left(0_{h, \tilde{g}}\right)=\tilde{g}^{k}\left(0_{h, \tilde{g}}\right)
$$

for $h, k \in \mathbb{Z}$. For $k=1$ this follows from $\mathrm{f})_{j+1}$ as $0_{h, \tilde{g}} \notin \operatorname{Int}\left(U_{k, \tilde{g}}\right)$ for each $k \in \mathbb{Z}$. For $k \in \mathbb{N}$ use a recursive argument, and finally, the case $-k$ follows from the case $k$. In particular, for each $h \in \mathbb{Z}, 0_{h, \tilde{g}}=0_{h, G_{j+1, \zeta}}$. It also follows that $G_{j+1, \zeta}^{q^{\prime}}(0)=\tilde{g}^{q^{\prime}}(0)=p^{\prime}$, hence $G_{j+1, \zeta}$ is of $\left(q, q^{\prime}, p, p^{\prime}\right)$ type and a $)_{j+1}$ and 1) $j+1$ easily follow.

Using 1$)_{j}$, for every $x \in U_{h, \tilde{g}}$ we have $x_{k, G_{j+1, \zeta}} \in U_{h+k, \tilde{g}}$ for $h, k \in \mathbb{Z}$. In particular, $\bar{z}_{k, G_{j+1, \zeta}}^{\prime} \in U_{1+k, \tilde{g}}$. Next, for $j>0$ we have $G_{j+1, \zeta}^{j q-1}\left(\bar{z}^{\prime}\right)=G_{j, \zeta}^{j q-1}\left(\bar{z}^{\prime}\right)$ as the $\left(j q-2, G_{j, \zeta}\right)$-orbit of $\bar{z}^{\prime}$ does not meet $U_{j q, \tilde{g}}$. It follows $G_{j+1, \zeta}^{j q}\left(\bar{z}^{\prime}\right)=$ 
$G_{j, \zeta}^{j q}\left(\bar{z}^{\prime}\right)-\zeta \bar{u}_{1}$. Moreover, even for $j=0$, using a similar argument we have $G_{j+1, \zeta}^{j q+q}\left(\bar{z}^{\prime}\right)=\tilde{g}^{q}\left(G_{j+1, \zeta}^{j q}\left(\bar{z}^{\prime}\right)\right)$, hence we get d $)_{j+1}$.

Finally, I prove the third step. Suppose that h) ${ }_{j}$ holds for every $j=0,1, \ldots, M_{1}$. Then, by the first and the second step, a $\left.\left.\left.\left.)_{j}, \mathrm{~b}\right)_{j} \mathrm{c}\right)_{j}, \mathrm{~d}\right)_{j}, \mathrm{f}\right)_{j}$ hold for $j=0,1, \ldots, M_{1}$ and e) $j$ holds for $j=0,1, \ldots, M_{1}-1$. We have for $0<j<M_{1}$

$$
G_{j+1, \zeta}^{j q}\left(\bar{z}^{\prime}\right) \leq G_{j+1, \zeta}\left(G_{j, \zeta}^{j q-1}\left(\bar{z}^{\prime}\right)\right)=G_{j, \zeta}^{j q}\left(\bar{z}^{\prime}\right)-\zeta \bar{u}_{1}
$$

I now prove

$$
G_{j, \hat{\varepsilon}}^{j q}\left(\bar{z}^{\prime}\right) \leq \tilde{g}^{j q}\left(\bar{z}^{\prime}\right)-\hat{\varepsilon} \bar{u}_{1} \sum_{h=1}^{j-1}\left(1-\omega_{1}\right)^{h}
$$

for $0<j \leq M_{1}$. Note that (8.2) is trivial for $j=1$. Suppose it holds for $0<j \leq$ $M_{1}-1$ and prove that it holds for $j+1$ as well. We have

$$
G_{j+1, \hat{\varepsilon}}^{(j+1) q}\left(\bar{z}^{\prime}\right) \leq G_{j+1, \hat{\varepsilon}}^{q}\left(G_{j, \hat{\varepsilon}}^{j q}\left(\bar{z}^{\prime}\right)-\hat{\varepsilon} \bar{u}_{1}\right) \leq G_{j+1, \hat{\varepsilon}}^{q}\left(c_{j}\right) \leq \tilde{g}^{q}\left(c_{j}\right)
$$

where $c_{j}=\tilde{g}^{j q}\left(\bar{z}^{\prime}\right)-\hat{\varepsilon} \bar{u}_{1} \sum_{h=0}^{j-1}\left(1-\omega_{1}\right)^{h}$. Since $0_{q+1, \tilde{g}} \leq 0_{(j+1) q+1, \tilde{g}} \leq \bar{z}_{(j+1) q-q^{\prime}, \tilde{g}}^{\prime}$, we have $c_{j} \geq 0_{1, \tilde{g}}+j p$, as, in the contrary case using Remark 4.3, we have

$$
G_{j+1, \hat{\varepsilon}}^{(j+1) q}\left(\bar{z}^{\prime}\right)<\tilde{g}^{q}\left(0_{1, \tilde{g}}+j p\right)=0_{q+1, \tilde{g}}+(j+1) p \leq \bar{z}_{(j+1) q-q^{\prime}, \tilde{g}}^{\prime}+(j+1) p
$$

contrary to $\mathrm{h})_{j+1}$. We deduce

$0_{1, \tilde{g}}+j p \leq c_{j} \leq \tilde{g}^{j q}\left(\bar{z}^{\prime}\right)=j p+\bar{z}_{j q, \tilde{g}}^{\prime}<0_{(j+1) q+1, \hat{g}}+j p<0_{\left(M_{1}+1\right) q+1, \hat{g}+j p}$.

Using Lemma 7.8 and (8.3) we deduce (8.2) for $j+1$. Using (8.2), Lemma 7.9ii and $(8.1)$, as $\left.\left[\tilde{g}^{j q}\left(\bar{z}^{\prime}\right)\right]=\left[\tilde{g}^{j q}\left(0_{1, \tilde{g}}\right)\right]=j p, \mathrm{~h}\right)_{j}$ does not hold for $j=M_{1}$.

Remark 8.2. It follows from 1$)_{j}$ in Lemma 8.1 and its proof that for $j=0, \ldots, \bar{j}$, $h \in \mathbb{Z}$, we have $U_{h, \tilde{g}}=U_{h, G_{j, \zeta}}$, and for each $k, r \in \mathbb{Z}, \tilde{g}^{k}(x)>0_{h, \tilde{g}}+r$ if and only if $G_{j, \zeta}^{k}(x)>0_{h, \tilde{g}}+r$, and the analogs for $<$ and $=$ in place of $>$ hold. Also, as $G_{j, \zeta} \leq \tilde{g}$, we have $x_{k, G_{j, \zeta}} \leq x_{k, \tilde{g}}$ for each $x \in[0,1[$ and $k \in \mathbb{N}$.

Lemma 8.3. $G_{\bar{j}, \hat{\varepsilon}}^{q^{\prime}}\left(\bar{z}^{\prime}\right)<\bar{z}^{\prime}+p^{\prime}$.

Proof. Composing the two hands of the equation in Lemma 8.1g) with $G_{\bar{j}, \hat{\varepsilon}}^{q^{\prime}-\bar{j} q}$, we get

$$
G_{\bar{j}, \hat{\varepsilon}}^{q^{\prime}}\left(\bar{z}^{\prime}\right)<\bar{z}^{\prime}+\bar{j} p-\left[\tilde{g}^{\bar{j} q-q^{\prime}}\left(\bar{z}^{\prime}\right)\right]
$$

and, as $\bar{z}^{\prime} \in U_{1, \tilde{g}}$ and $q^{\prime}>\bar{j} q+1$, using Lemma 4.2vii and Remark 4.3, we get

$$
\left[\tilde{g}^{\bar{j} q-q^{\prime}}\left(\bar{z}^{\prime}\right)\right]=\left[\tilde{g}^{\bar{j} q-q^{\prime}}\left(0_{1, \tilde{g}}\right)\right]=\left[\tilde{g}^{\bar{j} q}\left(0_{1, \tilde{g}}\right)-p^{\prime}\right]=\bar{j} p-p^{\prime}
$$


I now define $G$. Put

$$
\begin{aligned}
& \tilde{J}=\tilde{J}_{\zeta, \tau}=\left[\bar{z}_{-q^{\prime}, \tilde{g}}^{\prime}-2 V_{2} \bar{u}_{1}, \bar{z}^{\prime}+2 V_{2} \bar{u}_{1}\right], \\
& C=\left\{\zeta \in[0, \hat{\varepsilon}]: G_{\bar{j}_{, \zeta}}^{q^{\prime}}(x) \geq x+p^{\prime} \quad \forall x \in \tilde{J}\right\}, \\
& \bar{\zeta}=\bar{\zeta}_{\zeta, \tau}=\sup C, G=G_{\bar{j}, \bar{\zeta}} .
\end{aligned}
$$

Note that $0 \in C, \hat{\varepsilon} \notin C$. Note also that $G$ depends on $\varsigma, \tau$, and $\hat{\varepsilon}$. However, I will not mark this dependence and I will mean that $\varsigma, \tau$, and $\hat{\varepsilon}$ are fixed numbers satisfying the previous assumptions. I am now going to prove that $G$, unlike $\tilde{g}$ and $G_{j, \zeta}$, is sf-stable.

Lemma 8.4. If $x \in U_{1, \tilde{g}} \backslash \operatorname{Int}(\tilde{J})$ then $G^{h}(x)=\tilde{g}^{h}(x)$ for each $h=0, \ldots, q^{\prime}$.

Proof. Sketch. Note that by Lemma $8.1 \mathrm{e}_{j},\left[\gamma_{j}(\zeta)-\bar{u}_{1}, \gamma_{j}(\zeta)+\bar{u}_{1}\right] \subseteq \operatorname{Int}\left(U_{j q, \tilde{g}}\right)$ when $0 \leq j<\bar{j}$. It follows that the $\left(q^{\prime}-1, \tilde{g}\right)$-orbit of $x$ does not meet $\left[\gamma_{j}(\bar{\zeta})-\right.$ $\left.\bar{u}_{1}, \gamma_{j}(\bar{\zeta})+\bar{u}_{1}\right]$, the only nontrivial cases being $x_{i, \tilde{g}}$ with $i=q^{\prime}-1, j=0$, and $i=j q-1$. For the former case it suffices to observe that, in view of Corollary 4.6,

$$
\left|x_{q^{\prime}-1, \tilde{g}}-\bar{z}_{-1, \tilde{g}}^{\prime}\right| \geq \frac{1}{V_{2}}\left|x_{-1, \tilde{g}}-\bar{z}_{-1-q^{\prime}, \tilde{g}}^{\prime}\right| \geq \frac{2}{3 V_{2}}\left|x-\bar{z}_{-q^{\prime}, \tilde{g}}^{\prime}\right|>\bar{u}_{1},
$$

for the latter, note that using Lemma $8.1 \mathrm{e}_{j}$ again and Lemma 7.8,

$$
\begin{gathered}
\text { either } x_{j q-1, \tilde{g}} \geq \bar{z}_{j q-1, \tilde{g}}^{\prime}+V_{2} \bar{u}_{1} \geq \gamma_{j}(\bar{\zeta})+V_{2} \bar{u}_{1}>\gamma_{j}(\bar{\zeta})+\bar{u}_{1}, \\
\text { or } x_{j q-1, \tilde{g}}<\bar{z}_{j q-1-q^{\prime}, \tilde{g}}^{\prime}-\bar{u}_{1} \leq \gamma_{j}(\bar{\zeta})-\bar{u}_{1} .
\end{gathered}
$$

In conclusion, $G_{j, \bar{\zeta}}^{h}(x)=\tilde{g}^{h}(x)$ for each $h=0, \ldots, q^{\prime}$.

Corollary 8.5. $G$ is $\left(q, q^{\prime}, p, p^{\prime}\right)$ sf-stable, more precisely, there exists $\bar{z}_{1}^{\prime} \in \tilde{J} \subseteq$ $\operatorname{Int}\left(U_{1, \tilde{g}}\right)$ such that $G^{q^{\prime}}\left(\bar{z}_{1}^{\prime}\right)=\bar{z}_{1}^{\prime}+p^{\prime}$.

Proof. I prove i) in Definition 5.3. If $x \in U_{1, \tilde{g}} \backslash \tilde{J}$, then, in view of Lemma 8.4 , we have $G^{q^{\prime}}(x) \geq x+p^{\prime}$. The same inequality holds for $x \in \tilde{J}$, by the definition of $G$. As every $x \in \mathbb{R}$ has the form $k+G^{h}(y)$ for some $k, h$ integers and $\left.y \in U_{1, \tilde{g}}=U_{1, G}, \mathrm{i}\right)$ in Definition 5.3 holds for each real $x$. As $\hat{\varepsilon} \notin C$, then $\bar{\zeta}<\hat{\varepsilon}$, thus, by the definition of $\bar{\zeta}$, there exists $\bar{z}_{1}^{\prime} \in \tilde{J}$ such that $G^{q^{\prime}}\left(\bar{z}_{1}^{\prime}\right)=\bar{z}_{1}^{\prime}+p^{\prime}$. The inclusion $\tilde{J} \subseteq \operatorname{Int}\left(U_{1, \tilde{g}}\right)$ follows from Lemma 7.9i.

Corollary 8.6. $\alpha^{\prime}<\left(\bar{z}_{1}^{\prime}\right)_{-1, G}$. Moreover, $\alpha_{h-q^{\prime}, \tilde{g}}^{\prime} \leq \alpha_{h-q^{\prime}, G}^{\prime}$ for every $h=$ $1, \ldots, q^{\prime}$.

Proof. The first statement follows from Lemma 7.9i and the definition of $\tilde{J}$, and the second follows from Remark 8.2, as $h-q^{\prime} \leq 0$. 


\section{Proof of Lemma 5.5}

In this section I prove Lemma 5.5. I first prove that $G^{q}-I d-p$, which is positive as $\rho(G)>\frac{p}{q}$, is in fact greater than a positive constant only depending on $\zeta$ and not on $\tau$, at points whose $(q-1, G)$-orbit does not meet $\tilde{I}$. Consequently (Lemma 9.4), the $\left(q^{\prime}-1, G\right)$-orbit of $x$ meets $\tilde{I}$ on every interval of length $q$ apart from a set having cardinality bounded by a value depending on $\varsigma$ but not on $\tau$, and since $q^{\prime} \rightarrow+\infty$ as $\tau \rightarrow 0$, such a set is negligible for small $\tau$. Thanks to (7.2), this will allow us to prove the analog of $(5.3)$ for $(G, \Phi)$.

Here and in the following, we assume that $\hat{\varepsilon}$ is sufficiently small.

Lemma 9.1. There exists $\left.H_{1}(\varsigma) \in\right] 0,1[$ such that if the $(q-1, G)$-orbit of $x$ does not intersect $\tilde{I}$, then

$$
G^{q}(x) \geq x+p+H_{1}(\varsigma) .
$$

Proof. By Corollary 7.6 and Lemma 8.1i, for sufficiently small $\varsigma$ and $\hat{\varepsilon}$, it suffices to prove (9.1) with $\hat{g}$ in place of $G$ and $2 H_{1}(\varsigma)$ in place of $H_{1}(\varsigma)$. Moreover, the $(q-1, \hat{g})$-orbit of $x$ does not intersect $\left[\bar{z}_{1}-\sigma, \bar{z}_{1}+\sigma\right]$. As $\rho\left(\hat{g}_{\zeta, \zeta}\right)>\frac{p}{q}$, we have $\hat{g}_{\zeta, \zeta}^{q}(x)>x+p$ for each $x$, and by a compactness argument, there exists $H_{1}(\varsigma)>0$ such that $\hat{g}_{\zeta, \zeta}^{q}(x) \geq x+p+2 H_{1}(\varsigma)$ for each $x$. On the other hand, by the definition of $\hat{g}_{\zeta, \tau}, \hat{g}_{\zeta, \tau}(x)$ does not depend on $\tau$ if $x \in\left[0,1\left[\backslash \tilde{I}\right.\right.$, hence $\hat{g}_{\zeta, \zeta}^{q}(x)=\hat{g}_{\zeta, \tau}^{q}(x)$, so that we get (9.1) with $\hat{g}$ in place of $G$, and $2 H_{1}(\varsigma)$ in place of $H_{1}(\varsigma)$.

Corollary 9.2. We have $\mu_{h, G} \geq \frac{H_{1}(\varsigma)}{2^{|h|}}$ for each $h \in \mathbb{Z}$.

Proof. Using Corollary 7.4iii we have $\mu_{h, G} \geq \frac{1}{2^{|h|}} 0_{q, G}$. On the other hand, for $h=1, \ldots, q-1$, we have either $0_{h, g} \leq 0_{1, g}<\bar{z}_{1}-2 \sigma$ or $0_{h, g} \geq 0_{m+1, g}>$ $\bar{z}_{1}+2 \sigma$. Hence, for small $\varsigma$, thanks to Corollary 7.6, $0_{h, G} \notin \tilde{I}$ for $h=0, \ldots, q-1$. Therefore, by Lemma 9.1, $0_{q, G} \geq H_{1}(\varsigma)$.

For $x \in[0,1[$ put

$$
\mathcal{H}_{x}\left(=\mathcal{H}_{x, \zeta, \tau}\right)=\left\{h \in\left[0, q^{\prime}-1\right] \cap \mathbb{Z}: x_{h, G} \in \tilde{I}\right\} .
$$

Set $\mathcal{H}_{x}=\left\{h_{i, x}, i=1, \ldots, d_{x}\right\} \quad$ with $h_{1, x}<h_{2, x}<\ldots<h_{d_{x}, x}\left(d_{x}=0\right.$ if $\left.\mathcal{H}_{x}=\varnothing\right)$.

Lemma 9.3. Suppose $\varnothing \neq K \subseteq U_{k, g}, k \in \mathbb{Z}, K$ compact. Then, for $\varsigma$ and $\omega$ positive and sufficiently small, the image of the set $K_{\omega} \cap[0,1[$ via the maps $x \mapsto x_{h, G}$ are mutually disjoint for $h=0, \ldots, q-1$.

Proof. Consider the projection $\pi$ of $\mathbb{R}$ over the circle. As the sets $\pi\left(g^{h}(K)\right)$ are compact and contained in $\pi\left(U_{h+k, g}\right)$, which are mutually disjoint, the conclusion follows by a continuity argument. 
Lemma 9.4. Let $x \in[0,1[$. Then

i) $h_{i+1, x}-h_{i, x} \geq q$ for each $i=1, \ldots, d_{x}-1$.

ii) Let $D_{x}\left(=D_{x, \varsigma, \tau}\right)=\left[0, q^{\prime}-1\right] \cap \mathbb{Z} \backslash\left(\bigcup_{i=1}^{d_{x}-1}\left[h_{i, x}, h_{i, x}+q-1\right]\right)$. Then

$$
\#\left(D_{x}\right) \leq \frac{q}{H_{1}(\varsigma)}+2 q=: H_{2}(\varsigma) .
$$

\section{Proof.}

i) This follows from Lemma 9.3 with $\tilde{I}$ in place of $K$.

ii) I will prove that $\# D_{x}^{\prime} \leq \frac{1}{H_{1}(\zeta)}$, where $D_{x}^{\prime}=D_{x} \backslash\left([0, q-1] \cup\left[h_{d_{x}, x}, h_{d_{x}, x}+\right.\right.$ $q-1])$. Note that, by Lemma 9.1, we have

$$
\begin{gathered}
G^{h}(x)-\left(G^{h-q}(x)+p\right) \geq H_{1}(\varsigma) \text { if } h-1 \in D_{x}^{\prime}, \\
G^{h}(x)-\left(G^{h-q}(x)+p\right)>0 \text { in any case. }
\end{gathered}
$$

Given $k=0, \ldots, q-1$, let $u_{k} \in \mathbb{Z}$ be such that $q^{\prime}-q \leq k+u_{k} q \leq q^{\prime}-1$. Setting $D_{x, k}^{\prime}=D_{x}^{\prime} \cap\{k+r q: r \in \mathbb{Z}\}$, we have

$$
G^{k+1+u_{k} q}(x)-\left(G^{k+1}(x)+u_{k} p\right) \geq \# D_{x, k}^{\prime} H_{1}(\varsigma) .
$$

On the other hand, as $\rho(G)=\frac{p^{\prime}}{q^{\prime}}=\frac{p}{q}+\frac{1}{q q^{\prime}}<\frac{u_{k} p+1}{u_{k} q}$, we have $G^{k+1+u_{k} q}(x)-$ $\left(G^{k+1}(x)+u_{k} p\right)<1$. In conclusion, \# $D_{x, k}^{\prime} \leq \frac{1}{H_{1}(\varsigma)}$ and $\# D_{x}^{\prime} \leq \frac{q}{H_{1}(\varsigma)}$.

Next, I estimate the measure of some special sets. First, in Lemma 9.5, I prove that the function $G$ I will construct satisfies (5.4) (for $\left(q, q^{\prime}, p, p^{\prime}\right)$ type functions), then I define the set $J^{\prime}$ with respect to which $(G, \Phi)$ satisfies the definition of an oscillating pair. Such a set is a modification of the set defined in (5.4) suitable for $G$.

Lemma 9.5. $\mu\left(\bigcup_{h=1}^{q^{\prime}}\left[0_{h, G}, \alpha_{h-q^{\prime}, G}^{\prime}\right]\right)>1-\delta^{\prime}$.

Proof. Let $B$ be the complement in [0,1[ of the set in brackets. Then, also using Lemma 4.5 and the second inequality in (7.9), we get

$$
\frac{0_{q+1, \hat{g}}-\alpha_{1-q^{\prime}, \tilde{g}}^{\prime}}{\mu_{1, \tilde{g}}}=\frac{0_{q+1-q^{\prime}, \hat{g}}-\alpha_{1-q^{\prime}, \tilde{g}}^{\prime}}{\mu_{1, \tilde{g}}} \leq V_{2} \frac{0_{q+1, \hat{g}}-\alpha_{1, \tilde{g}}^{\prime}}{\mu_{1, \tilde{g}}}<\frac{\delta^{\prime}}{V_{2}}
$$

so that by Lemma 4.5 again, Lemma 4.2iv and Corollary 8.6, we get

$$
\mu(B) \leq \sum_{h=0}^{q^{\prime}-1}\left(0_{q+1+h, \hat{g}}-\alpha_{-q^{\prime}+1+h, \tilde{g}}^{\prime}\right) \leq \frac{\left(0_{q+1, \hat{g}}-\alpha_{1-q^{\prime}, \tilde{g}}^{\prime}\right) V_{2}}{\mu_{1, \tilde{g}}} \sum_{h=0}^{q^{\prime}-1} \mu_{h+1, \tilde{g}}<\delta^{\prime} .
$$


Let

$$
J^{\prime}=\bigcup_{h=1}^{q} \widetilde{D}_{h}, \quad \widetilde{D}_{h}:=\left[0_{h, g} \vee 0_{h, \tilde{g}},\left(\bar{z}_{2}\right)_{h-1, g}\right]
$$

Lemma 9.6. We have $\mu\left(J^{\prime}\right)>1-\delta_{1}^{\prime}$.

Proof. In view of (7.4) and Corollary 7.6ii, it suffices to prove that, for small $\varsigma$, $\left[\tilde{g}^{q}(0)\right]=\left[g^{q}(0)\right]$. Now, observe that $\tilde{g}^{q}(0)>p=g^{q}(0)$.

Next, I give some estimates for $x_{h, G}$. I will prove in Lemma 9.9, that when $x \in J^{\prime}$, then $x_{h, G}$ is sufficiently far from 0 and 1 , so that $\frac{1}{\Phi}$ has a good bound there, and, on the other hand, it is "frequently" in $\tilde{I}$. Then, in Lemma 9.10, I will prove a similar inequality for $x \in \tilde{J}$. I start with some preliminary results, concerning estimates of $x_{h, G}$ for special values of $h$. Note that the upper bound for $x_{h, G}$ in Lemma 9.9 is independent of $\varsigma, \tau$, and the one in Lemma 9.10 only depends on $\varsigma$.

Lemma 9.7. If $x \in \widetilde{D}_{h}, h=1, \ldots, q, k=0, \ldots, q-h+1$, then

$0_{h+k, G}+\left[g^{h+k}(0)\right]-\left[g^{h}(0)\right] \leq G^{k}(x)<\left(\bar{z}_{1}-2 \sigma\right)_{h+k-1, g}+\left[g^{h+k}(0)\right]-\left[g^{h}(0)\right]$.

Proof. We have

$$
g^{k}\left(\left(\bar{z}_{2}\right)_{h-1, g}\right)=\left(\bar{z}_{2}\right)_{h+k-1, g}+\left[g^{k}\left(0_{h, g}\right)\right]=\left(\bar{z}_{2}\right)_{h+k-1, g}+\left[g^{h+k}(0)\right]-\left[g^{h}(0)\right]
$$

and as for $x \in \widetilde{D}_{h}$ we have $G^{k}(x) \leq G^{k}\left(\left(\bar{z}_{2}\right)_{h-1, g}\right) \leq \tilde{g}^{k}\left(\left(\bar{z}_{2}\right)_{h-1, g}\right)$ and $\left(\bar{z}_{2}\right)_{h+k-1, g}<\left(\bar{z}_{1}-2 \sigma\right)_{h+k-1, g}$, for small $\varsigma$ we get the second inequality. To prove the first, observe that

$$
G^{k}(x) \geq G^{k}\left(0_{h, \tilde{g}}\right)=\tilde{g}^{k}\left(0_{h, \tilde{g}}\right)=0_{h+k, \tilde{g}}+\left[\tilde{g}^{h+k}(0)\right]-\left[\tilde{g}^{h}(0)\right]
$$

and that, for small $\varsigma$ we have $\left[\tilde{g}^{h+k}(0)\right]=\left[g^{h+k}(0)\right],\left[\tilde{g}^{h}(0)\right]=\left[g^{h}(0)\right]$; this can be seen as in the proof of Lemma 9.6.

Lemma 9.8. If $h>\frac{1}{H_{1}(\varsigma)}$ then $G^{h q}\left(0_{q+1, G}\right) \geq h p+\bar{z}_{1}-2 \sigma$.

Proof. Let $K:=\left[0_{1, g}, \bar{z}_{1}+2 \sigma\right] \subseteq U_{1, g}, U:=\left[0_{q+1, G}, \bar{z}_{1}-2 \sigma\left[\right.\right.$. As $\rho(G)>\frac{p}{q}$, then $G^{r q}(y)>y+r p$ for each $y \in \mathbb{R}$ and $r=1,2,3, \ldots$ For small $\varsigma$, we have $U \subseteq K_{\omega} \cap[0,1[$ where $\omega$ is so small that we can apply Lemma 9.3, thus for any $y \in U$ the $(q-1, G)$-orbit of $y$ does not intersect $\tilde{I}$, thus $G^{q}(y) \geq y+p+H_{1}(\varsigma)$ by Lemma 9.1. We easily deduce the assertion of the lemma. 
Lemma 9.9. Given a sufficiently small $\varsigma$, fix $M_{2} \in \mathbb{N} \backslash\{0,1\}$. Then there exists $\bar{\tau}_{\varsigma}>0$ such that if $0<\tau<\bar{\tau}_{\varsigma}$

i) For every $x \in\left[0_{q+1, G},\left(\bar{z}_{1}-2 \sigma\right)_{q, g}\left[\right.\right.$, for $0 \leq h \leq M_{2}$ we have

$$
h p+0_{q+1, G} \leq G^{h q}(x)<h p+\bar{z}_{1}+2 \sigma .
$$

ii) If $x \in J^{\prime}, k=0, \ldots, M_{2} q$, we have

$$
\alpha^{\prime}<0_{q, G} \leq x_{k, G} \leq \bar{v}_{1}:=\max _{r=0, \ldots, q} \frac{1+\left(\bar{z}_{1}+2 \sigma\right)_{r, g}}{2}<1 .
$$

\section{Proof.}

i) By Lemma 7.1, $\hat{g}_{\zeta, 0}^{h q}\left(\bar{z}_{1}\right)=\bar{z}_{1}+h p$ for every natural $h$, and for small $\varsigma$ we have $\left[0_{q+1, G},\left(\bar{z}_{1}-2 \sigma\right)_{q, g}\left[\subseteq\left[0_{q+1, G}, \bar{z}_{1}-\bar{a}_{5, \tau}[\right.\right.\right.$, independently of $\tau$, and by a continuity argument and the definition of $\tilde{g}$ and Remark 8.2, we conclude the proof of i).

ii) Let

$$
x \in \widetilde{D}_{h}, h=1, \ldots, q .
$$

If $k \leq q-h+1$ the result follows from Lemma 9.7. Using Lemma 9.7 with $k=q-h+1$ and i), we have $x_{q-h+1+d q, G} \in S:=\left[0_{q+1, G}, \bar{z}_{1}+2 \sigma[\right.$ for $d=0, \ldots, M_{2}$. It remains to prove

$$
0_{q, G} \leq y_{r, G} \leq \bar{v}_{1} \quad \forall y \in S \quad \forall r=1, \ldots, q-1 .
$$

To this aim, note that, for small $\zeta$, for such $r, 0_{-r, \tilde{g}} \notin \bar{S}$. In fact, $0_{1-q, \tilde{g}}<$ $0_{q+1, \tilde{g}}$, and, if $r<q-1$, then we have $-r \not \equiv 1 \bmod q$. On the other hand, (for small $\varsigma$ ) for any $h \in \mathbb{Z}$, then $0_{h, g} \in \bar{S}$ only (possibly) if $0_{h, g}=0_{1, g}$, that is if $h \equiv 1 \bmod q$. Hence $0_{-r, g} \notin \bar{S}$, and by continuity $0_{-r, \tilde{g}} \notin \bar{S}$. It follows that $\tilde{g}^{r}(S) \cap \mathbb{Z}=\varnothing$, and, for every $y \in S, 0_{q, G} \leq 0_{q+1+r, \tilde{g}} \leq y_{r, \tilde{g}} \leq\left(\bar{z}_{1}+2 \sigma\right)_{r, \tilde{g}}$. We conclude using Corollary 7.6ii) and Remark 8.2.

Lemma 9.10. Let $x \in \tilde{J}$. Then for all $k=0, \ldots, q^{\prime}$ we have

$$
\alpha^{\prime}<x_{k, G} \leq \bar{v}_{2}\left(=\bar{v}_{2}(\varsigma)\right):=1-\frac{H_{1}(\varsigma)}{2^{q} V_{2}} \frac{0_{q+1, \hat{g}}-\bar{z}^{\prime}}{\mu_{1, \tilde{g}}}<1 .
$$

Proof. I prove the first inequality. If $k \neq q^{\prime}-1$, then $x_{k, G} \notin U_{0, G}$, so that $x_{k, G} \geq$ $0_{q, \tilde{g}}>\alpha^{\prime}$. Suppose $k=q^{\prime}-1$. We have $x_{q^{\prime}-1, G} \geq \bar{x}_{q^{\prime}-1, G}=\bar{x}_{q^{\prime}-1, \tilde{g}}$ by 
Lemma 8.4, where $\bar{x}=\bar{z}_{-q^{\prime}, \tilde{g}}^{\prime}-2 V_{2} \bar{u}_{1}$. We thus have to prove $\bar{x}_{q^{\prime}-1, \tilde{g}}>\alpha^{\prime}$. We have

$$
\begin{aligned}
0_{q, \hat{g}}-\bar{x}_{q^{\prime}-1, \tilde{g}} & =0_{q^{\prime}+q, \hat{g}}-\bar{x}_{q^{\prime}-1, \tilde{g}} \\
& \leq \frac{\mu_{q^{\prime}, \tilde{g}}}{\mu_{1, \tilde{g}}} V_{2}\left(0_{q+1, \hat{g}}-\bar{x}\right) \\
& \leq 2 V_{2}\left(0_{q+1, \hat{g}}-\bar{x}\right) \leq 3 V_{2}^{2}\left(0_{q+1, \hat{g}}-\bar{z}^{\prime}\right) \\
& <\frac{2}{3}\left(0_{q+1, \hat{g}}-\alpha_{1, \tilde{g}}^{\prime}\right)<0_{q, \hat{g}}-\alpha^{\prime}
\end{aligned}
$$

where I have used Lemma 4.5ii, (7.11), Lemma 7.9i, (7.9) and the fact that $\mu_{q^{\prime}, \tilde{g}}=$ $\mu_{0, \tilde{g}} \leq \mu_{1, \tilde{g}} \max \frac{1}{\tilde{g}^{\prime}}$. For the second inequality note that $x_{k, G} \leq x_{k, \tilde{g}} \leq \tilde{x}_{k, \tilde{g}}$ where $\tilde{x}=\bar{z}^{\prime}+2 V_{2} \bar{u}_{1}$, and $\max _{k=0, \ldots, q^{\prime}} \tilde{x}_{k, \tilde{g}}=\tilde{x}_{q^{\prime}-q-1, \tilde{g}}$. On the other hand, by Lemma 4.5ii again and Corollary 9.2,

$$
1-\tilde{x}_{q^{\prime}-q-1, \tilde{g}} \geq \frac{\mu_{-q, \tilde{g}}}{\mu_{1, \tilde{g}}} \frac{1}{V_{2}}\left(0_{q+1, \hat{g}}-\bar{z}^{\prime}\right) \geq \frac{H_{1}(\varsigma)\left(0_{q+1, \hat{g}}-\bar{z}^{\prime}\right)}{2^{q} V_{2} \mu_{1, \tilde{g}}} .
$$

Finally, recall that $\frac{0_{q+1, \hat{\tilde{g}}}-\bar{z}^{\prime}}{\mu_{1, \tilde{g}}}$ is independent of $\varsigma$ and $\tau$. This has been noted in the considerations after Corollary 7.7. Hence $\bar{v}_{2}$ only depends on $\varsigma$ and not on $\tau$.

So far, we have only investigated $G$. In order to prove Lemma 5.5 we now need also to study $\Phi$. Let

$$
\begin{gathered}
\bar{u}_{2}=\frac{\alpha+\left(\bar{z}_{2}\right)_{q-1, g}}{2}, \quad \bar{u}_{3}=\frac{\alpha-\left(\bar{z}_{2}\right)_{q-1, g}}{2}>0, \\
\bar{\eta}=\min \left\{\eta, \frac{1}{\Lambda^{\prime} q}\right\} .
\end{gathered}
$$

Define $(\Phi=) \Phi_{\eta^{\prime}}:\left[\alpha^{\prime}, 1\right] \rightarrow \mathbb{R}$ for $\left.\eta^{\prime} \in\right] 0, \bar{\eta}[$ by

$$
\Phi(x)= \begin{cases}\hat{\psi}\left(\frac{x-\bar{u}_{2}}{\bar{u}_{3}}\right)\left(\eta^{\prime}-\eta\right)+\eta & \text { if } x \in\left[\alpha^{\prime}, \alpha[\right. \\ \phi(x) & \text { if } x \in[\alpha, 1] .\end{cases}
$$

Lemma 9.11. $\Phi$ is a $C^{\infty} \alpha^{\prime}$-caliber extending $\left.\phi, \alpha^{\prime} \in\right] 0, \frac{\alpha}{2}\left[\right.$. Moreover, $\Phi=\eta^{\prime}$ on $\left[\alpha^{\prime}, \bar{u}_{2}\right] \supseteq\left[\alpha^{\prime}, 0_{q, \tilde{g}}\right], \Phi \geq \eta^{\prime}$ and $\left|\Phi^{(h)}\right| \leq L_{h} \eta \frac{1}{\bar{u}_{3}^{h}}$ on $\left.] \alpha^{\prime}, \alpha\right]$. Finally, $\bar{u}_{3}$ does not depend on $\eta^{\prime}$.

Proof. Note that for small $\varsigma$ we have $p<\tilde{g}^{q}(0)<p+\frac{\bar{u}_{2}}{2}$ so that $0<\alpha^{\prime}<0_{q, \tilde{g}}<$ $\frac{\bar{u}_{2}}{2}<\frac{\alpha}{2}<\alpha<1$. The Lemma easily follows. 
In the following proposition I study the oscillation properties of $\Phi$.

Proposition 9.12. There exists $\tau_{\varsigma}>0$ such that if $0<\tau<\tau_{\varsigma}$ then

i) $T_{\Phi}(G, q, x)>\Lambda^{\prime} q$ for each $x \in J^{\prime}$,

ii) There exists $w^{\prime}:=M_{2} q$ with $M_{2}>1, M_{2}$ integer, $M_{2} q<q^{\prime}$, such that $T_{\Phi}\left(G, w^{\prime}, x\right)<\lambda w^{\prime}$ for each $x \in J^{\prime}$,

iii) $T_{\Phi}\left(G, q^{\prime}, x\right)<\lambda q^{\prime}$ for each $x \in \tilde{J}$.

Proof. For given $\varsigma>0$, choose $M_{2}$ sufficiently large (see proof of ii)) and suppose $0<\tau<\bar{\tau}_{\varsigma}$, so that we can apply Lemma 9.9.

i) $J^{\prime} \subseteq \mathcal{O}\left(G, M_{2} q, \alpha^{\prime}\right)$ by Lemma 9.9. Let now $x \in \widetilde{D}_{h}, h=1, \ldots, q$. A simple verification shows that

$$
g^{q-h}(x) \geq p-\left[g^{h}(0)\right], \quad \tilde{g}^{q-h}(x) \geq p-\left[\tilde{g}^{h}(0)\right]
$$

so that for small $\varsigma, \tilde{g}^{q-h}(x) \geq p-\left[g^{h}(0)\right]$. Moreover, $g^{q-h}(x) \leq p-$ $\left[g^{h}(0)\right]+\left(\bar{z}_{2}\right)_{q-1, g}$. Thus, since $\left(\bar{z}_{2}\right)_{q-1, g}<\bar{u}_{2}$, then $x_{q-h, G} \leq x_{q-h, \tilde{g}}<\bar{u}_{2}$, hence

$$
T_{\Phi}(G, q, x) \geq \frac{1}{\Phi\left(x_{q-h, G}\right)}=\frac{1}{\eta^{\prime}}>\Lambda^{\prime} q .
$$

ii) Let $x \in \widetilde{D}_{h}, h=1, \ldots, q$. Define

$$
k_{j}= \begin{cases}0 & \text { if } j=0 \\ q-h+(j-1) q+1 & \text { if } j=1, \ldots, n-1 \\ n q & \text { if } j=n\end{cases}
$$

where $n=M_{2}$. We have $x_{k_{1}, G} \in\left[0_{q+1, G},\left(\bar{z}_{1}-2 \sigma\right)_{q, g}[\right.$ by Lemma 9.7, hence by Lemmas 9.8 and $9.9 \mathrm{i}$ we have

$$
x_{k_{j}, G} \in \tilde{I} \text { if } n>j>H_{3}(\varsigma):=2+\left[\frac{1}{H_{1}(\varsigma)}\right] \text {. }
$$

Put $\hat{\eta}_{1}\left(=\hat{\eta}_{1}(\varsigma)\right):=\min \left\{\eta^{\prime}, \min _{\left[\alpha, \bar{v}_{1}\right]} \phi\right\}>0$, so that, by Lemma 9.9ii, $\Phi\left(x_{i, G}\right) \geq$ $\hat{\eta}_{1}$ for each $i=0, \ldots, M_{2} q$. Now, using (7.2) and a continuity argument, there exists $M^{\prime}<\lambda q$ such that

$$
T_{\Phi}(G, q, x) \leq M^{\prime} \forall x \in \tilde{I}
$$


with $M^{\prime}$ independent of $\varsigma$ (sufficiently small) and $\tau$. Hence supposing $M_{2} \geq$ $H_{3}(\varsigma)+3$, we get

$$
\begin{aligned}
T_{\Phi}\left(G, M_{2} q, x\right)= & \sum_{j=0}^{n-1} T_{\Phi}\left(G, k_{j+1}-k_{j}, x_{k_{j}, G}\right) \\
= & \left(\sum_{j=0}^{H_{3}(\varsigma)} T_{\Phi}\left(G, k_{j+1}-k_{j}, x_{k_{j}, G}\right)\right) \\
& +\left(\sum_{j=H_{3}(\varsigma)+1}^{n-2} T_{\Phi}\left(G, q, x_{k_{j}, G}\right)\right) \\
& +T_{\Phi}\left(G, k_{n}-k_{n-1}, x_{k_{n-1}, G}\right) \\
\leq & \left(H_{3}(\varsigma)+1\right) \frac{q}{\hat{\eta}_{1}}+M_{2} M^{\prime}+\frac{2 q}{\hat{\eta}_{1}} .
\end{aligned}
$$

Since $M^{\prime}<\lambda q$, for sufficiently large $M_{2}$, say $M_{2}>H_{4}(\varsigma)$, we have $T_{\Phi}\left(G, M_{2} q, x\right)<\lambda M_{2} q$.

iii) In view of Lemma 9.4, using the definition of $D_{x}$, for $q^{\prime}>H_{2}(\varsigma)$ we have $d_{x} \geq 2$ and $q^{\prime}=\#\left(D_{x}\right)+\left(d_{x}-1\right) q$. Thus, using Lemma 9.10 and (9.2),

$$
\begin{aligned}
T_{\Phi}\left(G, q^{\prime}, x\right) & =\sum_{k \in D_{x}} \frac{1}{\Phi\left(x_{k, G}\right)}+\sum_{i=1}^{d_{x}-1} \sum_{k=h_{i, x}}^{h_{i, x}+q-1} \frac{1}{\Phi\left(x_{k, G}\right)} \\
& \leq \frac{H_{2}(\varsigma)}{\hat{\eta}_{2}}+\sum_{i=1}^{d_{x}-1} T_{\Phi}\left(G, q, x_{h_{i, x}, G}\right) \\
& \leq \frac{H_{2}(\varsigma)}{\hat{\eta}_{2}}+\left(d_{x}-1\right) M^{\prime},
\end{aligned}
$$

where $\hat{\eta}_{2}\left(=\hat{\eta}_{2}(\varsigma)\right):=\min \left\{\eta^{\prime}, \min _{\left[\alpha, \bar{v}_{2}\right]} \phi\right\}>0$. Thus, if $q^{\prime}$ is greater than a constant $H_{5}(\varsigma)$, then iii) holds. In conclusion, in order that i), ii) and iii) hold, we have to take $M_{2}>H_{4}(\varsigma)$, then $\tau_{\varsigma}=\min \left\{\bar{\tau}_{\varsigma}, \tau\left(H_{5}(\varsigma), \varsigma\right)\right\}$ (see Corollary 7.5ii).

Proof of Lemma 5.5. See Lemma 9.5, Corollary 8.5, Lemma 9.11, Lemma 9.6, Proposition 9.12, Lemma 8.1. 


\section{References}

[1] Y. BRENIER, Remarks on some linear hyperbolic equations with oscillatory coefficients, In: "Third International Conference on Hyperbolic Problems" (Uppsala 1990), Studentlitteratur, Lund, 1991, 119-130.

[2] I. P. CORNFELD, S. V. Fomin and YA. G. SinAI, "Ergodic Theory," Springer-Verlag, New York, 1982.

[3] E. DE Giorgi, On the convergence of solutions of some evolution differential equations, Set-Valued Anal. 2 (1994), 175-182.

[4] W. E., Homogenization of linear and nonlinear transport equations, Comm. Pure Appl. Math. 45 (1992), 301-326.

[5] T. Y. HoU and X. XIN, Homogenization of linear transport equations with oscillatory vector fields, SIAM J. Appl. Math. 52 (1992), 34-45.

[6] A. KatoK and B. Hasselblatt, "Introduction to the Modern Theory of Dynamical Systems", Cambridge University Press, Cambridge, 1995.

[7] M. MisiurewiCZ and K. Ziemian, Rotation sets for Maps of Tori, J. London Math. Soc. (2) 40 (1989), 490-506.

[8] R. PEIRONe, Homogenization of ordinary and linear transport equations, Differential Integral Equations 9 (1996), 323-334.

[9] R. PeIRone, Convergence of solutions of linear transport equations, Ergodic Theory Dynam. Systems 23 (2003), 919-933.

[10] T. TASSA, Homogenization of two-dimensional linear flows with integral invariance, SIAM J. Appl. Math. 57 (1997), 1390-1405.

Università di Roma "Tor Vergata" Dipartimento di Matematica Via della Ricerca Scientifica 00133, Roma, Italia peirone@mat.uniroma2.it 